T. Okada

Nagoya Math. J.

Vol. 194 (2009), 1-32

\title{
NONRATIONAL WEIGHTED HYPERSURFACES
}

\author{
TAKUZO OKADA
}

\begin{abstract}
The aim of this paper is to construct (i) infinitely many families of nonrational $\mathbb{Q}$-Fano varieties of arbitrary dimension $\geq 4$ with at most quotient singularities, and (ii) twelve families of nonrational $\mathbb{Q}$-Fano threefolds with at most terminal singularities among which two are new and the remaining ten give an alternate proof of nonrationality to known examples. These are constructed as weighted hypersurfaces with the reduction mod $p$ method introduced by Kollár [10].
\end{abstract}

\section{$\S 1$. Introduction}

We say that a normal projective variety defined over the field $\mathbb{C}$ of complex numbers is a $\mathbb{Q}$-Fano variety if its anticanonical divisor is an ample $\mathbb{Q}$-Cartier divisor. A terminal (resp. log terminal) $\mathbb{Q}$-Fano variety is a $\mathbb{Q}$ Fano variety with at most terminal (resp. log terminal) singularities.

$(\mathbb{Q}-)$ Fano varieties were originally studied as candidates for nonrational unirational varieties, that is, counterexamples to the Lüroth problem. In the early seventies, V. A. Iskovskikh-Ju. I. Manin [8], and H. ClememsP. Griffith [4] independently proved the nonrationality of smooth quartic threefolds and smooth cubic threefolds respectively by developing different mothods, while all the smooth cubic threefolds and a certain smooth quartic threefold have been known to be unirational.

The rationality problem is quite subtle in dimension $>3$ as well. Essentially there are only three known methods to construct nonrational varieties of dimension $>3$ which are either unirational or $(\mathbb{Q}$-)Fano. Firstly, M. ArtinD. Mumford [1] constructed a unirational smooth threefold $X$ with nonzero torsion in $H^{3}(X, \mathbb{Z})$ so that $X \times \mathbb{P}^{n-3}$ is a nonrational unirational $n$-fold for each $n \geq 3$. Secondly, A. V. Pukhlikov (e.g. [14]) proved the nonrationality of general Fano hypersurfaces of dimension $n$ and degree $n+1$ with $n \geq 4$

Received December 22, 2007.

Accepted June 6, 2008.

2000 Mathematics Subject Classification: Primary 14E08, 14J45; Secondary 14J30, $14 \mathrm{~J} 70$.

The author is supported by JSPS Research Fellowships for Young Scientists. 
by studying their birational self map groups (the method initiated by [8]). Thirdly, Kollár [10] proved the nonrationality of very general Fano hypersurfaces of dimension $n \geq 3$ and degree $d$ such that $n \geq d \geq 2\lceil(n+3) / 3\rceil$. We refer the readers to J. Kollár [11, V.5] for a more detailed account.

In this paper, we apply Kollár's techniques [10] to weighted hypersurfaces. In general, weighted hypersurfaces have singularities and this makes our proof complicated. As a result, we obtain nonrational terminal $\mathbb{Q}$-Fano threefolds and infinitely many families of nonrational log terminal $\mathbb{Q}$-Fano varieties in each dimension $\geq 4$ (cf. Section 7 ). We note that the examples constructed in this paper are all rationally connected because a log terminal $\mathbb{Q}$-Fano variety is rationally connected [15].

Definition 1.1. Let $X$ be a variety of dimension $n$ over a field $k$.

- We say that $X$ is rational if there is a birational map $\mathbb{P}_{k}^{n} \rightarrow-\rightarrow$.

- We say that $X$ is ruled (resp. uniruled) if there is a variety $Y$ of dimension $n-1$ over $k$ and a birational map (resp. dominant rational map) $Y \times \mathbb{P}_{k}^{1} \rightarrow X$.

- In positive characteristics, we say that $X$ is separably uniruled if the above rational map $Y \times \mathbb{P}_{k}^{1} \rightarrow X$ is also separable.

- Let $\bar{k}$ be an algebraic closure of $k$. We say that $X$ is geometrically ruled if $X_{\bar{k}}=X \times_{\operatorname{Spec} k} \operatorname{Spec} \bar{k}$ is ruled.

We work over the weighted projective space $\mathbb{P}\left(1, a_{1}, \ldots, a_{n}, b\right)$ with homogeneous coordinates $x_{0}, \ldots, x_{n}$ and $y$. Definitions and some basic properties of weighted projective spaces will be treated in the next section. For a graded ring $S$ and homogeneous elements $f_{1}, \ldots, f_{m} \in S$, by

$$
\left(f_{1}=\cdots=f_{m}=0\right) \subset \operatorname{Proj} S
$$

we mean the closed subscheme defined by the homogeneous ideal $\left(f_{1}, \ldots\right.$, $\left.f_{m}\right)$ of $S$. In the same way, for a $\operatorname{ring} A$ and elements $f_{1}, \ldots, f_{m} \in A$,

$$
\left(f_{1}=\cdots=f_{m}=0\right) \subset \operatorname{Spec} A
$$

denote the closed subscheme defined by the ideal $\left(f_{1}, \ldots, f_{m}\right)$ of $A$.

Now we state the main theorems. Condition 2.1 and 2.3 in the statement below are introduced in Section 2 . 
TheOREM 1.2. Assume that $\left(p,\left\{a_{i}\right\}, b, n, d\right)$ satisfies Condition 2.1 and 2.3. Then, the weighted hypersurface

$$
X_{f}:=\left(y^{p} x_{0}-f\left(x_{0}, \ldots, x_{n}\right)=0\right) \subset \mathbb{P}_{\mathbb{C}}\left(1, a_{1}, \ldots, a_{n}, b\right)
$$

of degree $d$ is a non-ruled log terminal $\mathbb{Q}$-Fano variety of dimension $n$ for a very general $f=f\left(x_{0}, \ldots, x_{n}\right) \in H_{d}(\mathbb{C})$.

Here, for a field $k$, we denote by $H_{d}(k)$ the $k$-vector space $k\left[x_{0}, \ldots, x_{n}\right]_{d}$, the degree $d$ part of the graded ring $k\left[x_{0}, \ldots, x_{n}\right]$ whose grading is given by $\operatorname{deg} x_{i}=a_{i}$. By convention we say that $f$ is very general when it does not belong to countable union of suitable proper closed subvarieties.

TheOREM 1.3. Assume that $\left(p,\left\{a_{i}\right\}, b, n, d\right)$ satisfies Condition 2.1 and 2.3. Let $\mathbb{k}$ be an algebraically closed field of characteristic $p$. Then, the weighted hypersurface

$$
X_{f}:=\left(y^{p} x_{0}-f\left(x_{0}, \ldots, x_{n}\right)=0\right) \subset \mathbb{P}_{\mathbb{k}}\left(1, a_{1}, \ldots, a_{n}, b\right)
$$

is not separably uniruled for a general $f=f\left(x_{0}, \ldots, x_{n}\right) \in H_{d}(\mathbb{k})$.

Remark 1.4. It is originally proved in [10] that a certain $p$-fold covering of a smooth variety is not separably uniruled in characteristic $p$. Specifically, it is proved that, under certain conditions on positive integers $p, a$ and $n$, a general weighted hypersurface of the form

$$
X=\left(y^{p}-f\left(x_{0}, \ldots, x_{n}\right)=0\right) \subset \mathbb{P}(1, \ldots, 1, a)
$$

is nonrational, where $\operatorname{deg} x_{i}=1, \operatorname{deg} y=a$ and $\operatorname{deg} f=p a$.

The point of this paper is to treat various kinds of weights and allow the projection map $X_{f} \rightarrow \mathbb{P}\left(1, a_{1}, \ldots, a_{n}\right)$ to have a point of indeterminacy.

The following result of Matsusaka enables us to pass to positive characteristics where we can make use of the unusual behavior of differential forms.

Theorem 1.5. ([13], Appendix, Theorem 1.1, [11], IV, Theorem 1.6) Let $R$ be an excellent discrete valuation ring and $X$ a normal irreducible scheme. Let $T$ be Spec $R$ and $\varphi: X \rightarrow T$ a proper surjective morphism with connected fibers. Then the following assertions hold. 
(1) If the generic fiber of $\varphi$ is ruled over the quotient field of $R$, then every irreducible component of the special fiber of $\varphi$ is ruled over the residue field of $R$.

(2) If the generic fiber of $\varphi$ is geometrically ruled, then every reduced irreducible component of the special fiber of $\varphi$ is geometrically ruled.

Lemma 1.6. ([10], Lemma 7) Let $X$ be a smooth proper variety and $\mathcal{M}$ a big line bundle on $X$. Assume that there is an injection $\mathcal{M} \hookrightarrow \Omega_{X}^{i}$ for some $i>0$. Then $X$ is not separably uniruled.

For a line bundle $\mathcal{L}$ on a normal projective variety $X$, we say that $\mathcal{L}$ is big if some positive multiple of $\mathcal{L}$ defines a birational map onto its image.

Lemma 1.6 is a key to the proof of Theorem 1.3. Since Lemma 1.6 is only valid for smooth varieties, we construct a desingularization $\varphi: Y \rightarrow X_{f}$ in Section 3. In Section 4 and 5, we construct a big line bundle on $Y$ which is contained in $\Omega_{Y}^{n-1}$. Section 6 consists of the proof of Theorem 1.2 and 1.3. Some of the examples which are obtained by Theorem 1.2 are presented in Section 7 .

Notation And Terminology. Throughout this paper, $p>1$ is a prime number. We denote by $\mathbb{C}$ the field of complex numbers and by $\mathbb{k}$ an algebraically closed field of characteristic $p$. Let us fix some notation with respect to group schemes. Let $k$ be a field.

- $\mathbb{G}_{\mathrm{m}, k}$ is the one dimensional torus $\operatorname{Spec} k\left[t, t^{-1}\right]$ and we write $\mathbb{G}_{\mathrm{m}}$ instead of $\mathbb{G}_{\mathrm{m}, \mathbb{k}}$.

- For a positive integer $r$, we denote by $\boldsymbol{\mu}_{r, k}$ the finite group scheme Spec $k[t] /\left(t^{r}-1\right)$. We write $\boldsymbol{\mu}_{r}$ instead of $\boldsymbol{\mu}_{r, \mathbb{k}}$.

- Let $A$ be a $k$-algebra of finite type and $X$ the affine scheme $\operatorname{Spec} A$. Let $G=\operatorname{Spec} R$ be an affine group scheme over $k$.

Suppose we are given an action of $G$ on $X$. There is a homomorphism $\phi: A \rightarrow A \otimes_{k} R$ of $k$-algebras which in turn induces the given action. We write $A^{G}$ for the ring of invariants $\{g \in A \mid \phi(g)=g \otimes 1\} \subset A$. If $A$ is generated by $z_{1}, \ldots, z_{n}$ as a $k$-algebra and $\phi$ is determined by sending $z_{i}$ to $w_{i} \in A \otimes_{k} R$, we say that $G$ acts on $X$ by $z_{i} \mapsto w_{i}$.

We frequently consider the following action: Put $A=k\left[x_{1}, \ldots, x_{n}\right]$ and $R=k\left[t, t^{-1}\right]$ (resp. $k[t] /\left(t^{r}-1\right)$ ). Let $\alpha_{1}, \ldots, \alpha_{n}$ be non-negative integers and let $\phi: A \rightarrow A \otimes_{k} R$ be the homomorphism of $k$-algebras 
determined by sending $x_{i}$ to $x_{i} \otimes t^{\alpha_{i}}$ (resp. $\left.x_{i} \otimes \bar{t}^{\alpha_{i}}\right)$. Then, the induced morphism $G \times \mathbb{A}^{n} \rightarrow \mathbb{A}^{n}$ of schemes defines an action of $G$ on $\mathbb{A}^{n}$. In this case, we say that $\mathbb{G}_{\mathrm{m}, k}\left(\right.$ resp. $\left.\boldsymbol{\mu}_{r, k}\right)$ acts on $\mathbb{A}^{n}$ by $x_{i} \mapsto x_{i} \otimes t^{\alpha_{i}}$ (resp. $x_{i} \mapsto x_{i} \otimes \bar{t}^{\alpha_{i}}$ ).

- Let $(X, x)$ be a germ of a variety of dimension $n$ over a field $\mathbb{C}$. We say that the singularity of $X$ at $x$ is of type $\frac{1}{r}\left(\alpha_{1}, \ldots, \alpha_{n}\right)$ if $(X, x)$ is analytically isomorphic to $\left(\mathbb{A}^{n} / \boldsymbol{\mu}_{r, \mathbb{C}}, o\right)$, where $o$ is the image of the origin and the $\boldsymbol{\mu}_{r, \mathbb{C}}$ action on $\mathbb{A}^{n}$, whose affine coordinates are $x_{1}, \ldots, x_{n}$, is given by $x_{i} \mapsto x_{i} \otimes \bar{t}^{\alpha_{i}}$.

Acknowledgment. The author expresses his gratitude to Professor Shigefumi Mori for valuable comments and suggestions. The author is grateful to Professor Noboru Nakayama for pointing out many errors in the draft versions of this paper. The author is also grateful to Professors Shigeru Mukai and Masayuki Kawakita for helpful discussions and warm encouragements. The author would like to thank Professor János Kollár whose comments on our earlier version motivated the author to improve the paper.

\section{$\S 2$. Conditions}

For a prime number $p$ and positive integers $n, d, a_{0}, \ldots, a_{n}, b$, we consider the following conditions.

\section{CONDition 2.1.}

(1) $n \geq 3$ and $a_{0}=1$.

(2) $d=p b+1$.

(3) $\operatorname{gcd}\left\{a_{1}, \ldots, a_{n}\right\}=1$ and there are at least two $i$ among $1, \ldots, n$ such that $a_{i}$ is coprime to $p$.

(4) $\sum_{i=0}^{n} a_{i}<d<\sum_{i=0}^{n} a_{i}+b$.

(5) For any algebraically closed field $\mathbb{k}$ of characteristic $p$, a general weighted hypersurfaces of degree $d$ in $\mathbb{P}_{\mathbb{k}}\left(a_{1}, \ldots, a_{n}\right)$ is quasi smooth.

In the following, we define $a_{n+1}:=b$ and we sometimes write $a_{n+1}$ instead of writing $b$ for simplicity of the description. For a subset $I \subset$ $\{1, \ldots, n+1\}$, we define $r_{I}=\operatorname{gcd}\left\{a_{i} \mid i \in I\right\}$. A subset $I \subset\{1, \ldots, n+1\}$ 
is called saturated if $\operatorname{gcd}\left\{r_{I}, a_{i}\right\} \neq r_{I}$ for every $i \in\{1, \ldots, n+1\} \backslash I$. We define

$$
\mathcal{I}=\left\{I \subset\{1, \ldots, n+1\} \mid r_{I}>1 \text { and if } r_{I} \mid d \text { then }|I|>1\right\} .
$$

and

$$
\mathcal{I}_{\text {sat }}=\{I \in \mathcal{I} \mid I \text { is saturated }\} .
$$

For an integer $m \in \mathbb{Z}$ and a subset $I \subset\{1, \ldots, n+1\}$, we denote by $[m]^{I}$ the integer such that $m \equiv[m]^{I}\left(\bmod r_{I}\right)$ and $0 \leq[m]^{I}<r_{I}$.

Suppose that $\left(p,\left\{a_{i}\right\}, b, n, d\right)$ satisfies Condition 2.1. For $I \in \mathcal{I}_{\text {sat }}$, we define

$$
I^{\#}:= \begin{cases}\{0, \ldots, n\} \backslash I & \text { if } n+1 \notin I \text { and } r_{I} \mid d, \\ \{0, \ldots, n\} \backslash(I \cup\{i\}) & \text { if } n+1 \notin I \text { and } r_{I} \nmid d, \\ \{1, \ldots, n+1\} \backslash(I \cup\{i\}) & \text { if } n+1 \in I,\end{cases}
$$

where the $i$ in the second and the third cases is the minimum number such that $d-a_{i} \equiv 0\left(\bmod r_{I}\right)$. If $r_{I} \nmid d$, then Condition 2.1 ensures that there exists $i$ such that $d-a_{i} \equiv 0\left(\bmod r_{I}\right)$ (cf. Case 2 and Case 3 in the proof of Lemma 5.7). Hence, $I^{\#}$ is well-defined.

For $j=1, \ldots, n$, let $\psi_{j}^{\prime}:\{1, \ldots, b-1\} \rightarrow \mathbb{Z}^{n-1}$ be the map defined by

$$
\begin{aligned}
\psi_{j}^{\prime}(k):= & \left((1 / b)\left(a_{j}\left[k a_{1}\right]^{I}-a_{1}\left[k a_{j}\right]^{I}\right), \ldots,(1 / b)\left(a_{j}\left[k a_{j-1}\right]^{I}-a_{j-1}\left[k a_{j}\right]^{I}\right),\right. \\
& \left.(1 / b)\left(a_{j}\left[k a_{j+1}\right]^{I}-a_{j+1}\left[k a_{j}\right]^{I}\right), \ldots,(1 / b)\left(a_{j}\left[k a_{n}\right]^{I}-a_{n}\left[k a_{j}\right]^{I}\right)\right),
\end{aligned}
$$

where $I=\{n+1\}$, and $\psi_{j}:\{1, \ldots, b-1\} \rightarrow(\mathbb{Z} / p \mathbb{Z})^{n-1}$ be the composite of $\psi_{j}^{\prime}$ and the natural projection $\mathbb{Z}^{n-1} \rightarrow(\mathbb{Z} / p \mathbb{Z})^{n-1}$.

Definition 2.2. For $j=1, \ldots, n$, we denote by $\Psi_{j}$ the set $\{1, \ldots, b\} \backslash$ $\psi_{j}^{-1}(0)$.

For $\left(p,\left\{a_{i}\right\}, b, n, d\right)$ satisfying Condition 2.1, we consider the following additional condition.

Condition 2.3. Put $A=d-\sum_{i=0}^{n} a_{i}$.

(1) For any $I \in \mathcal{I}_{\text {sat }}$ with $I \neq\{n+1\}$, we have

$$
A>r_{I}-\min _{0<k<r_{I}}\left\{\sum_{i \in I^{\#}}\left[k a_{i}\right]^{I}\right\} \text {. }
$$

(2) For $I=\{n+1\}$, there exists some $j \in\{1, \ldots, n\}$ such that $p \nmid a_{j}$ and

$$
A>b-\min _{k \in \Psi_{j}}\left\{\sum_{i=1}^{n}\left[k a_{i}\right]^{I}\right\} .
$$




\section{$\S 3 . ~ S i n g u l a r i t i e s$ and desingularization}

First we recall the definition of weighted projective space and study its basic properties. For details, we refer the reader to [6].

Definition 3.1. Let $c_{0}, \ldots, c_{n}$ be positive integers and $k$ a field. The weighted projective space $\mathbb{P}_{k}\left(c_{0}, \ldots, c_{n}\right)$ over $k$ is defined by

$$
\mathbb{P}_{k}\left(c_{0}, \ldots, c_{n}\right)=\operatorname{Proj} k\left[x_{0}, \ldots, x_{n}\right],
$$

where $k\left[x_{0}, \ldots, x_{n}\right]$ is the graded polynomial ring with $\operatorname{deg} x_{i}=c_{i}$. The variables $x_{0}, \ldots, x_{n}$ are called homogeneous coordinates.

Note that the one dimensional torus $\mathbb{G}_{\mathrm{m}, k}$ acts on $\mathbb{A}_{k}^{n+1}$ by $x_{i} \mapsto x_{i} \otimes t^{c_{i}}$ and then $\mathbb{P}_{k}\left(c_{0}, \ldots, c_{n}\right)$ is the geometric quotient $\left(\mathbb{A}_{k}^{n+1} \backslash\{0\}\right) / \mathbb{G}_{\mathrm{m}, k}$. The affine piece $\mathrm{D}_{+}\left(x_{i}\right)=\left(x_{i} \neq 0\right)$ is isomorphic to $\mathbb{A}_{k}^{n} / \boldsymbol{\mu}_{c_{i}, k}$, where $\boldsymbol{\mu}_{c_{i}, k}$ acts on $\mathbb{A}_{k}^{n}$ by $\xi_{j} \mapsto \xi_{j} \otimes \bar{t}^{c_{j}}$ for all $j \neq i$ on the coordinates $\left\{\xi_{0}, \ldots, \hat{\xi}_{i}, \ldots, \xi_{n}\right\}$ of $\mathbb{A}_{k}^{n}$. The variable $\xi_{j}$ is identified with $x_{j} x_{i}^{-c_{j} / c_{i}}$.

Definition-Lemma 3.2. Let $X$ be a closed subscheme of the weighted projective space $\mathbb{P}=\mathbb{P}_{k}\left(c_{0}, \ldots, c_{n}\right)$ and let $\tau: \mathbb{A}_{k}^{n+1} \backslash\{0\} \rightarrow \mathbb{P}$ the canonical morphism. The punctured affine cone $C_{X}^{*}$ of $X$ is defined by $C_{X}^{*}=\tau^{-1}(X)$ and the affine cone $C_{X}$ is the scheme-theoretic closure of $C_{X}^{*}$ in $\mathbb{A}_{k}^{n+1}$.

For a subset $I \subset\{0, \ldots, n\}$, we denote by $\mathrm{D}_{+}\left(x_{I}\right)$ the affine open subset $\mathrm{D}_{+}\left(\prod_{i \in I} x_{i}\right)$ of $\mathbb{P}$. We define

$$
\begin{aligned}
C_{\mathbb{P}, I} & :=\operatorname{Spec} k\left[\left\{\xi_{i} \mid i \in\{0, \ldots, n\} \backslash I\right\}\right] \times \operatorname{Spec} k\left[u_{1}, u_{1}^{-1}, \ldots, u_{|I|-1}, u_{|I|-1}^{-1}\right] \\
& \cong \mathbb{A}_{k}^{n+1-|I|} \times\left(\mathbb{A}_{k}^{1} \backslash\{0\}\right)^{|I|-1}
\end{aligned}
$$

Put $F=\prod_{i \in I} x_{i}^{\alpha_{i}}$, where $\left\{\alpha_{i} \mid i \in I\right\}$ are integers such that $\sum_{i \in I} \alpha_{i} c_{i}=$ $\operatorname{gcd}\left\{c_{i} \mid i \in I\right\}=: r$. We identify $\xi_{i}$ with $x_{i} F^{-a_{i} / r}$ for $i \notin I$ and $u_{j}$ with $x_{j}^{a_{j_{0}, j}} x_{j_{0}}^{-a_{j_{0}, j}}$ for $j=1, \ldots,|I|-1$, where $j_{0}$ is the minimum of $I$ and $a_{i, j}=$ $a_{i} / \operatorname{gcd}\left\{a_{i}, a_{j}\right\}$. This yields a natural morphism $\tau_{I}: C_{\mathbb{P}, I} \rightarrow \mathrm{D}_{+}\left(x_{I}\right)$ and an isomorphism $C_{\mathbb{P}, I} / \boldsymbol{\mu}_{r, k} \cong \mathrm{D}_{+}\left(x_{I}\right)$, where the $\boldsymbol{\mu}_{r, k}$-action on $C_{\mathbb{P}, I}$ is defined by $\xi_{i} \mapsto \xi_{i} \otimes \bar{t}^{a_{i}}$ for $i \notin I$ and $u_{j} \mapsto u_{j}$ for $j=1, \ldots,|I|-1$. We define $C_{X, I}:=\tau_{I}^{-1}\left(X \cap \mathrm{D}_{+}\left(x_{I}\right)\right)$. By a slight abuse of notation, we denote by $\tau_{I}: C_{X, I} \rightarrow X \cap \mathrm{D}_{+}\left(x_{I}\right)$ the restriction of the morphism $C_{\mathbb{P}, I} \rightarrow \mathrm{D}_{+}\left(x_{I}\right)$. If $I=\{i\}$ then we write $C_{X, i}=C_{X, I}$ and $\tau_{i}=\tau_{I}$.

Note that $\mathbb{G}_{\mathrm{m}, k}$ (resp. $\boldsymbol{\mu}_{r, k}$ ) acts on $C_{X}^{*}$ (resp. $\left.C_{X, I}\right)$ and gives the isomorphism $X \cong C_{X}^{*} / \mathbb{G}_{\mathrm{m}, k}\left(\operatorname{resp} . X \cap \mathrm{D}_{+}\left(x_{I}\right) \cong C_{X, I} / \boldsymbol{\mu}_{r, k}\right)$. 
The proof is straightforward and we leave it to the reader.

Definition 3.3. A closed subscheme $X$ in $\mathbb{P}_{k}\left(c_{0}, \ldots, c_{n}\right)$ is called quasi smooth if its affine cone $C_{X}$ is smooth outside the origin.

In this paper, we consider weighted projective spaces $\mathbb{P}\left(1, a_{1}, \ldots, a_{n}, b\right)$ with homogeneous coordinates $x_{0}, \ldots, x_{n}, y$ and $\mathbb{P}\left(1, a_{1}, \ldots, a_{n}\right)$ with homogeneous coordinates $x_{0}, \ldots, x_{n}$.

Definition 3.4. Suppose that $\left(p,\left\{a_{i}\right\}, b, n, d\right)$ satisfies Condition 2.1. For a field (or more generally a ring) $k$, we denote by $H_{d}(k)$ the $k$-vector space (or $k$-module) $k\left[x_{0}, \ldots, x_{n}\right]_{d}$, the degree $d$ part of the graded ring $k\left[x_{0}, \ldots, x_{n}\right]$ whose grading is given by $\operatorname{deg} x_{i}=a_{i}$.

$H_{d}(k)$ can be naturally identified with a $k$-vector subspace of $H^{0}\left(\mathbb{P}_{k}(1\right.$, $\left.\left.a_{1}, \ldots, a_{n}, b\right), \mathcal{O}(d)\right)$. For $f=f\left(x_{0}, \ldots, x_{n}\right) \in H_{d}(k)$, set

$$
X_{f}:=\left(y^{p} x_{0}-f=0\right) \subset \mathbb{P}_{k}\left(1, a_{1}, \ldots, a_{n}, b\right) .
$$

Quasi smoothness is important since singularities of a quasi smooth variety are all caused by the $\mathbb{G}_{\mathrm{m}, k}$ action.

Lemma 3.5. Assume that $\left(p,\left\{a_{i}\right\}, b, n, d\right)$ satisfies Condition (2.1.5). Then, a general weighted hypersurface of degree $d$ in $\mathbb{P}_{\mathbb{C}}\left(a_{1}, \ldots, a_{n}\right)$ is quasi smooth.

Proof. Fix a general homogeneous polynomial $g \in \mathbb{C}\left[x_{1}, \ldots, x_{n}\right]$ of degree $d$, where the grading is given by $\operatorname{deg} x_{i}=a_{i}$. Let $R$ be a subring of $\mathbb{C}$ which is of finite type over $\mathbb{Z}$ such that $g \in R\left[x_{1}, \ldots, x_{n}\right]$ and the localization $R_{(p)}$ of $R$ at the ideal $(p)$ is a discrete valuation ring. Then, the geometric special fiber of $Z:=(g=0) \subset \mathbb{P}_{R_{(p)}}\left(a_{1}, \ldots, a_{n}\right)$ is smooth by Condition (2.1.5). Thus, there is a smooth weighted hypersurface of degree $d$ in $\mathbb{P}_{\mathbb{C}}\left(a_{1}, \ldots, a_{n}\right)$, which implies the smoothness of a general weighted hypersurface.

Lemma 3.6. Let $k=\mathbb{k}$ or $\mathbb{C}$, where $\mathbb{k}$ is an algebraically closed field of characteristic $p$. Assume that $\left(p,\left\{a_{i}\right\}, b, n, d\right)$ satisfies Condition 2.1. Then we have

$$
\operatorname{Sing}\left(C_{X_{f}}\right) \cap\left(x_{0}=0\right)=\{0\}
$$

for a general $f \in H_{d}(k)$. 
Proof. Put $X=X_{f}$. By the Jacobi criterion, we have

$$
\operatorname{Sing}\left(C_{X}\right)=\left(y^{p} x_{0}-f=y^{p}-\frac{\partial f}{\partial x_{0}}=\frac{\partial f}{\partial x_{1}}=\cdots=\frac{\partial f}{\partial x_{n}}=p y^{p-1} x_{0}=0\right) .
$$

If we write $f=f_{d}+f_{d-1} x_{0}+\cdots+f_{0} x_{0}^{d}$, where $f_{j}=f_{j}\left(x_{1}, \ldots, x_{n}\right)$ is a weighted homogeneous polynomial of degree $j$, we have

$$
\frac{\partial f}{\partial x_{i}}=\frac{\partial f_{d}}{\partial x_{i}}+\frac{\partial f_{d-1}}{\partial x_{i}} x_{0}+\cdots+\frac{\partial f_{1}}{\partial x_{i}} x_{0}^{d-1}
$$

for $i=1, \ldots, n$. Thus, we see that

$$
\begin{aligned}
& \operatorname{Sing}\left(C_{X}\right) \cap\left(x_{0}=0\right) \\
& =\left(x_{0}=y^{p}-f_{d-1}=0\right) \cap\left(f_{d}=\frac{\partial f_{d}}{\partial x_{1}}=\cdots=\frac{\partial f_{d}}{\partial x_{n}}=0\right) .
\end{aligned}
$$

Condition (2.1.5) or Lemma 3.5 implies that $\operatorname{Spec}\left(k\left[x_{1}, \ldots, x_{n}\right] /\left(f_{d}\right)\right)$ is smooth outside the origin. Thus, we have $\operatorname{Sing}\left(C_{X}\right) \cap\left(x_{0}=0\right)=\{0\}$.

\subsection{Quasi smoothness over $\mathbb{C}$}

Lemma 3.7. Assume that $\left(p,\left\{a_{i}\right\}, b, n, d\right)$ satisfies Condition 2.1 and $\mathbb{P}\left(1, a_{1}, \ldots, a_{n}, b\right)$ is defined over $\mathbb{C}$. Then $X_{f}$ is quasi smooth for a general $f \in H_{d}(\mathbb{C})$.

Proof. Put $X=X_{f}$. By Lemma 3.6, it suffices to show that $C_{X} \cap\left(x_{0} \neq\right.$ $0)$ is smooth. By the Jacobi criterion, we have

$$
\begin{aligned}
& \operatorname{Sing}\left(C_{X}\right) \cap\left(x_{0} \neq 0\right) \\
& =\left(\frac{\partial f}{\partial x_{0}}=\frac{\partial f}{\partial x_{1}}=\cdots=\frac{\partial f}{\partial x_{n}}=f=0\right) \cap(y=0) \cap\left(x_{0} \neq 0\right) .
\end{aligned}
$$

Hence, it suffices to show that a general weighted hypersurfaces of degree $d$ in $\mathbb{P}_{\mathbb{C}}\left(a_{0}, \ldots, a_{n}\right)$ is quasi smooth. This follows from the quasi smoothness criterion [7, Theorem 8.1] and Lemma 3.5.

\subsection{Construction of a desingularization of $X_{f}$ over $\mathbb{k}$}

Unfortunately, in positive characteristics, $X_{f}$ is not quasi smooth in general. Next, we consider singularities of $X_{f}$ which lie on $\mathrm{D}_{+}\left(x_{0}\right)$. Let us recall some definitions and basic properties of critical points. For proofs and details see $[11, \mathrm{IV}]$. 
Definition-Lemma 3.8. Let $X$ be a smooth variety of dimension $n$ over a field $k$ of characteristic $p$ and $f$ a function on $X$. Let $x \in X$ be a closed point and assume that $f$ has a critical point at $x$. Choose local coordinates $x_{1}, \ldots, x_{n}$ at $x$.

(1) The matrix $H(f)=\left(\partial^{2} f / \partial x_{i} \partial x_{j}\right)$ is called the Hessian of $f$.

(2) $f$ has a nondegenerate critical point at $x$ if $\operatorname{rank} H(f)(x)=\operatorname{dim} X$.

If $p \neq 2$ or $p=2$ and $n$ is even, then $f$ has a nondegenerate critical point at $x$ if and only if in suitable local coordinates $f$ can be written as

$$
f= \begin{cases}c+x_{1} x_{2}+\cdots+x_{n-1} x_{n}+f_{3} & \text { if } n \text { is even } \\ c+x_{1}^{2}+x_{2} x_{3}+\cdots+x_{n-1} x_{n}+f_{3} & \text { if } n \text { is odd }\end{cases}
$$

where $c \in k$ and $f_{3} \in \mathfrak{m}_{x}^{3}$.

(3) If $p=2$ and $\operatorname{dim} X$ is odd, then every critical point is degenerate.

(4) Assume that $p=2$ and $\operatorname{dim} X$ is odd. A critical point of $f$ is called almost nondegenerate if length $\mathcal{O}_{x, X} /\left(\partial f / \partial x_{1}, \ldots, \partial f / \partial x_{n}\right)=2$. Equivalently, in suitable local coordinates $f$ can be written as

$$
f=c+a x_{1}^{2}+x_{2} x_{3}+\cdots+x_{n-1} x_{n}+b x_{1}^{3}+f_{3},
$$

where $a, b, c \in k, b \neq 0, f_{3} \in \mathfrak{m}_{x}^{3}$ and the coefficient of $x_{1}^{3}$ in $f_{3}$ is 0 .

Throughout this subsection, we assume the following.

\section{Assumption 3.9 .}

- $\left(p,\left\{a_{i}\right\}, b, n, d\right)$ satisfies Condition 2.1.

- We work over an algebraically closed field $\mathbb{k}$ of characteristic $p$.

We denote by $U$ the affine open subset $\mathrm{D}_{+}\left(x_{0}\right)$ of $\mathbb{P}\left(1, a_{1}, \ldots, a_{n}, b\right)$. We see that $U$ is the affine space $\mathbb{A}^{n+1}$ with coordinates $\xi_{1}, \ldots, \xi_{n}$ and $\nu$, where we identify $\xi_{i}$ with $x_{i} / x_{0}^{a_{i}}$ for $i=1, \ldots, n$ and $\nu$ with $y / x_{0}^{b}$. Put $f^{\prime}=f / x_{0}^{d}=f\left(1, \xi_{1}, \ldots, \xi_{n}\right)$ for $f \in H_{d}(\mathbb{k})$. Then $X_{f} \cap U$ is defined by the equation $\nu^{p}-f^{\prime}=0$. Let $A$ be the polynomial ring $\mathbb{k}\left[\xi_{1}, \ldots, \xi_{n}\right]$ and consider the natural projection

$$
\psi: X_{f} \cap U=\operatorname{Spec}\left(A[\nu] /\left(\nu^{p}-f^{\prime}\right)\right) \longrightarrow \operatorname{Spec} A=: V .
$$

Lemma 3.10. Notation as above. Then $f^{\prime} \in A$ has only (almost) nondegenerate critical points on $V$ for a general $f \in H_{d}(\mathbb{k})$. 
Proof. For every closed point $v \in V$, the natural map $H_{d}(\mathbb{k}) \rightarrow$ $\mathcal{O}_{V, v} / \mathfrak{m}_{v}^{2}$ which maps $g$ to $\bar{g}^{\prime}$, where $g^{\prime}=g\left(1, \xi_{1}, \ldots, \xi_{n}\right)$, is surjective since $a_{i}<d$ for every $i$.

We show that, for every closed point $v \in V$, there is an element $g \in$ $H_{d}(\mathbb{k})$ which has an (almost) nondegenerate critical point at $v$. We may assume that $a_{1} \leq a_{i}$ for every $i$. Then, by Condition (2.1.1) and (2.1.4), we have $3 a_{1}<d$ and $a_{i}+a_{j}<d$ for every distinct $i, j$. Hence, if $n$ is even there exists $g_{1} \in H_{d}(\mathbb{k})$ such that

$g_{1}^{\prime}=\left(\xi_{1}-v_{1}\right)\left(\xi_{2}-v_{2}\right)+\left(\xi_{3}-v_{3}\right)\left(\xi_{4}-v_{v}\right)+\cdots+\left(\xi_{n-1}-v_{n-1}\right)\left(\xi_{n}-v_{n}\right)$,

and if $n$ is odd there exist $g_{2}, g_{3} \in H_{d}(\mathbb{k})$ such that

$$
\begin{aligned}
& g_{2}^{\prime}=\left(\xi_{1}-v_{1}\right)^{2}+\left(\xi_{2}-v_{2}\right)\left(\xi_{3}-v_{3}\right)+\left(\xi_{4}-v_{4}\right)\left(\xi_{5}-v_{5}\right) \\
& +\cdots+\left(\xi_{n-1}-v_{n-1}\right)\left(\xi_{n}-v_{n}\right) \\
& g_{3}^{\prime}=\left(\xi_{2}-v_{2}\right)\left(\xi_{3}-v_{3}\right)+\left(\xi_{4}-v_{4}\right)\left(\xi_{5}-v_{5}\right) \\
& +\cdots+\left(\xi_{n-1}-v_{n-1}\right)\left(\xi_{n}-v_{n}\right)+\left(\xi_{1}-v_{1}\right)^{3} .
\end{aligned}
$$

If $p \neq 2$ or $p=2$ and $n$ is even then $g_{1}$ or $g_{2}$ has nondegenerate critical point at $v$. If $p=2$ and $n$ is odd then $g_{3}$ has almost nondegenerate critical point at $v$.

Fix $v \in V$ and let $W_{v} \subset H_{d}(\mathbb{k})$ be the set of functions with a critical point at $v$. The codimension of $W_{v}$ in $H_{d}(\mathbb{k})$ is $n$. In $W_{v}$, the set of functions with an (almost) nondegenerate critical point at $v$ form an open set $W_{v}^{\circ}$ which is nonempty. Thus the set of functions with a degenerate critical point is $\bigcup_{v \in V}\left(W_{v} \backslash W_{v}^{\circ}\right)$ and it has codimension at least one in $H_{d}(\mathbb{k})$.

Lemma 3.11. Notation as above. Then $X_{f} \cap U$ has only isolated hypersurface singularities which can be resolved by successive blow ups at each singular points for a general $f \in H_{d}(\mathbb{k})$.

Proof. For an element $f \in H_{d}(\mathbb{k})$, we put $f^{\prime}=f\left(1, \xi_{1}, \ldots, \xi_{n}\right)$ and $X_{0}=X_{f} \cap \mathrm{D}_{+}\left(x_{0}\right)$. We see that

$$
X_{0}=\left(\nu^{p}-f^{\prime}=0\right) \subset \mathbb{A}^{n+1},
$$

and

$$
\operatorname{Sing}\left(X_{0}\right)=\left(\nu^{p}-f^{\prime}=\frac{\partial f^{\prime}}{\partial \xi_{1}}=\cdots=\frac{\partial f^{\prime}}{\partial \xi_{n}}=0\right)
$$


It follows from Lemma 3.10 that $f^{\prime}$ has only (almost) nondegenerate critical points on $V$ for a general $f \in H_{d}$. Thus, $X_{0}$ has only isolated singular points which correspond to critical points of $f^{\prime}$. It follows from [11, V, Proposition 5.10] that the isolated singular points of $X_{0}$ can be resolved by successive blow ups at each singular points.

Lemma 3.12. Fix a general $f \in H_{d}(\mathbb{k})$ and put

$$
X_{\mathrm{qs}}:=X \backslash \operatorname{Sing}\left(X \cap \mathrm{D}_{+}\left(x_{0}\right)\right), \quad U_{\mathrm{qs}}:=X_{\mathrm{qs}} \cap \mathrm{D}_{+}\left(x_{0} \cdots x_{n} y\right),
$$

where $X=X_{f}$. Then $U_{\mathrm{qs}} \subset X_{\mathrm{qs}}$ is a toroidal embedding without selfintersection.

Proof. We refer the readers to [9] for the definition of a toroidal embedding (without self-intersection). By Lemma 3.6, the punctured affine cone $C_{X_{\mathrm{qs}}}^{*}:=\tau^{-1}\left(X_{\mathrm{qs}}\right)$ is smooth and the singular locus of $X_{\mathrm{qs}}$ is contained in $X_{\mathrm{qs}} \backslash U_{\mathrm{qs}}$. Smoothness of $C_{X_{\mathrm{qs}}}^{*}$ implies that $U_{\mathrm{qs}} \subset X_{\mathrm{qs}}$ is a toroidal embedding (cf. Section 5.2). For each $i=0, \ldots, n$, the closed subscheme $X \cap\left(x_{i}=0\right)$ is isomorphic to a weighted hypersurface contained in $\mathbb{P}\left(a_{0}, \ldots, \hat{a}_{i}, \ldots, a_{n}, b\right)$ and, in particular, normal. Therefore, $U_{\mathrm{qs}} \subset X_{\mathrm{qs}}$ is a toroidal embedding without self-intersection.

Corollary 3.13. Let $f \in H_{d}(\mathbb{k})$ be a general element and put $X=$ $X_{f}$. There exists a desingularization $\varphi: Y \rightarrow X$ with the following propertie:

(1) Around the singular points on $X \cap \mathrm{D}_{+}\left(x_{0}\right), \varphi$ is the composition of blow ups at each singular points.

(2) The restriction $\varphi: \varphi^{-1}\left(X_{\mathrm{qs}}\right) \rightarrow X_{\mathrm{qs}}$ is a resolution of the toroidal embedding $U_{\mathrm{qs}} \subset X_{\mathrm{qs}}$.

Proof. This follows immediately from Lemma 3.11 and 3.12. We refer the reader to [9] for the existence of a desingularization of a toroidal embedding.

\section{$\S 4$. Construction of a big line bundle}

In the previous section, we show that there is a desingularization $\varphi: Y \rightarrow$ $X=X_{f}$. In this section, we construct a big line bundle on $Y$ which is contained in $\Omega_{Y}^{n-1}$. Throughout this section, we assume the following.

Assumption 4.1. 
- $\left(p,\left\{a_{i}\right\}, b, n, d\right)$ satisfies Condition 2.1 and 2.3.

- We work over an algebraically closed field $\mathbb{k}$ of characteristic $p$.

- The weighted homogeneous polynomial $f=f\left(x_{0}, \ldots, x_{n}\right)$ is a general element of $H_{d}(\mathbb{k})$ and $X=X_{f}$.

- We choose and fix a desingularization $\varphi: Y \rightarrow X$ which satisfies the properties (1) and (2) of Corollary 3.13.

- We denote by $A$ the integer

$$
A=d-\sum_{i=0}^{n} a_{i}
$$

There is a natural projection

$$
\pi: \mathbb{P}\left(1, a_{1}, \ldots, a_{n}, b\right) \backslash\{(0: \cdots: 0: 1)\} \longrightarrow \mathbb{P}\left(1, a_{1}, \ldots, a_{n}\right) .
$$

Let $V$ be the smooth locus of $\mathbb{P}\left(1, a_{1}, \ldots, a_{n}\right)$. Put $U=\pi^{-1}(V)$ and $X^{\circ}=$ $X \cap U$. By Condition (2.1.3), we see that $U$ is smooth and the codimension of $X \backslash X^{\circ}$ in $X$ is greater than or equal to 2. By a slight abuse of notation, the restriction of $\pi$ on $X^{\circ}$ is again denoted by $\pi: X^{\circ} \rightarrow V$.

For an integer $l$, we denoted by $\mathcal{O}_{X^{\circ}}(l)$ the restriction of the tautological sheaf $\mathcal{O}(l)$ of $\mathbb{P}\left(1, a_{1}, \ldots, a_{n}, b\right)$ on $X^{\circ}$. The sheaf $\mathcal{O}_{X^{\circ}}(l)$ is invertible on $X^{\circ}$ for every integer $l$ since $\mathcal{O}(l)$ is invertible on $U$.

\section{LEMMA 4.2. Notation as above.}

(1) There is an exact sequence; $\left.0 \rightarrow \pi^{*} \Omega_{V}^{1} \rightarrow \Omega_{U}^{1}\right|_{X^{\circ}} \rightarrow \mathcal{O}_{X^{\circ}}(-b) \rightarrow 0$.

(2) There is an exact sequence; $\left.0 \rightarrow \mathcal{O}_{X^{\circ}}(-d) \stackrel{\delta}{\rightarrow} \Omega_{U}^{1}\right|_{X^{\circ}} \rightarrow \Omega_{X^{\circ}}^{1} \rightarrow 0$, and we have $\operatorname{Im} \delta \subset \pi^{*} \Omega_{V}^{1}$.

(3) There is an exact sequence;

$$
0 \longrightarrow \operatorname{Coker}\left[\mathcal{O}_{X^{\circ}}(-d) \stackrel{\delta}{\rightarrow} \pi^{*} \Omega_{V}^{1}\right] \longrightarrow \Omega_{X^{\circ}}^{1} \longrightarrow \mathcal{O}_{X^{\circ}}(-b) \longrightarrow 0 .
$$

Proof. There is a locally splitting exact sequence

$$
0 \longrightarrow \pi^{*} \Omega_{V}^{1} \longrightarrow \Omega_{U}^{1} \longrightarrow \mathcal{O}_{U}(-b) \longrightarrow 0 \text {. }
$$

Pulling back this sequence to $X^{\circ}$ we obtain (1). The existence of the exact sequence of (2) is a general fact. (3) follows from (1) and (2). We check locally to see that $\operatorname{Im} \delta$ is contained in $\pi^{*} \Omega_{V}^{1}$. 
Take a point $u \in X^{\circ}$. We can choose local coordinates $z_{1}, \ldots, z_{n}, w$ of $U$ at $u$ so that $z_{1}, \ldots, z_{n}$ form local coordinates of $V$ at $\pi(u)$ and $X^{\circ}$ is defined by the equation $w^{p} g^{\prime}\left(z_{1}, \ldots, z_{n}\right)-f^{\prime}\left(z_{1}, \ldots, z_{n}\right)=0$ around $u$, where $g^{\prime}, f^{\prime}$ and $w$ correspond to $x_{0}, f$ and $y$ respectively. We see that $\operatorname{Im} \delta$ is generated by

$$
d\left(w^{p} g^{\prime}-f^{\prime}\right)=p w^{p-1} g^{\prime} d w+w^{p} d g^{\prime}-d f^{\prime}=w^{p} d g^{\prime}-d f^{\prime}
$$

and, thus, it is contained in $\pi^{*} \Omega_{V}^{1}$.

Notice that $X^{\circ}$ is not smooth in general. It may have isolated singular points on $X^{\circ} \cap \mathrm{D}_{+}\left(x_{0}\right)$ as described in Lemma 3.11. If we restrict the sequences in (1), (2) and (3) of Lemma 4.2 on the smooth locus of $X^{\circ}$, then those are exact sequences of locally free sheaves.

Definition 4.3. Let $\mathcal{M}^{\circ}$ be the double dual of

$$
\bigwedge^{n-1}\left(\operatorname{Coker}\left[\mathcal{O}_{X^{\circ}}(-d) \stackrel{\delta}{\rightarrow} \pi^{*} \Omega_{V}^{1}\right]\right)
$$

and $\mathcal{M}=i_{*} \mathcal{M}^{\circ}$, where $i: X^{\circ} \hookrightarrow X$ is the embedding. Let $M$ be a Weil divisor on $X$ such that $\mathcal{O}_{X}(M) \cong \mathcal{M}$.

Lemma 4.2 implies that

$$
\mathcal{M} \cong \mathcal{O}_{X}\left(d-\sum_{i=0}^{n} a_{i}\right)=\mathcal{O}_{X}(A)
$$

and $\mathcal{M} \subset\left(\Omega_{X}^{n-1}\right)^{\vee \vee}$. By Condition (2.1.4), $M$ is ample.

Let $F$ be the exceptional divisor of $\varphi: Y \rightarrow X$ which is obtained by resolving isolated singular points on $X \cap \mathrm{D}_{+}\left(x_{0}\right)$. Let $E$ be the exceptional divisor of $\varphi: Y \rightarrow X$ away from $F$, that is, $E$ is obtained by resolving the singularities of the toroidal embedding $U_{\mathrm{qs}} \subset X_{\mathrm{qs}}$ and then let $E=\bigcup_{i} E_{i}$ be the irreducible decomposition. The restriction of $\mathcal{O}_{Y}\left(\left\lfloor\varphi^{*} M\right\rfloor\right)$ on $Y \backslash(E \cup F)$ can be seen as a subsheaf of $\left.\Omega_{Y}^{n-1}\right|_{Y \backslash(E \cup F)}$.

Definition 4.4. For each $i$, let $\gamma_{i}$ be the largest integer such that $\mathcal{O}_{Y}\left(\left\lfloor\varphi^{*} M\right\rfloor+\gamma_{i} E_{i}\right)$ is contained in $\Omega_{Y}^{n-1}$ generically around $E_{i}$. We define $\mathcal{L}:=\mathcal{O}_{Y}\left(\left\lfloor\varphi^{*} M\right\rfloor+\sum \gamma_{i} E_{i}\right)$.

By the definition, we have $\left.\left.\mathcal{L}\right|_{Y \backslash F} \subset \Omega_{Y}^{n-1}\right|_{Y \backslash F}$.

LEMMA 4.5. $\mathcal{L}$ is a subsheaf of $\Omega_{Y}^{n-1}$. 
Proof. Put $X_{0}=X \cap \mathrm{D}_{+}\left(x_{0}\right), Y_{0}=\varphi^{-1}\left(X_{0}\right)$ and $\varphi_{0}=\left.\varphi\right|_{Y_{0}}: Y_{0} \rightarrow X_{0}$. We need to show that $\left.\mathcal{L}\right|_{Y_{0}}=\varphi_{0}^{*}\left(\left.\mathcal{M}\right|_{X_{0}}\right) \subset \Omega_{Y_{0}}^{n-1}$. The restriction of the projection

$$
\pi_{0}=\left.\pi\right|_{X_{0}}: X_{0} \rightarrow \mathrm{D}_{+}\left(x_{0}\right) \subset \mathbb{P}\left(1, a_{1}, \ldots, a_{n}\right)
$$

is identified with the morphism

$$
\operatorname{Spec} A[\nu] /\left(\nu^{p}-f^{\prime}\right) \longrightarrow \operatorname{Spec} A=\mathbb{A}^{n},
$$

where $A=\mathbb{k}\left[\xi_{1}, \ldots, \xi_{n}\right]$ and $f^{\prime}=f\left(1, \xi_{1}, \ldots, \xi_{n}\right) \in A$. Consider the homomorphism of $A$-modules $\rho_{f^{\prime}}: A \rightarrow \Omega_{A}^{1}$ determined by $\rho_{f^{\prime}}(1)=d f^{\prime}$. We have $\left.\delta\right|_{X_{0}}=-\pi_{0}^{*} \rho_{f^{\prime}}$ and this implies that $\left.\mathcal{M}\right|_{X_{0}}=\pi_{0}^{*} \mathcal{Q}$, where $\mathcal{Q}$ is the invertible sheaf on $\mathbb{A}^{n}$ associated with the $A$-module $\left(\bigwedge^{2} \operatorname{Coker}\left(\rho_{f^{\prime}}\right)\right)^{\vee \vee}$.

It is proved in [10] that the invertible sheaf $\pi_{0}^{*} \mathcal{Q}$ is generated by the $(n-1)$-form $\eta$ which is defined in Remark 4.6 below and that $\varphi_{0}^{*} \eta$ does not have a pole along exceptional divisors of $\varphi_{0}$ (cf. [10, Section 22, 23]). Therefore, we have $\left.\mathcal{L}\right|_{Y_{0}}=\varphi_{0}^{*}\left(\pi_{0}^{*} \mathcal{Q}\right) \subset \Omega_{Y_{0}}^{n-1}$.

Remark 4.6. It is shown in $\left[10\right.$, Lemma 16] that $\left.\mathcal{M}\right|_{X \cap D_{+}\left(x_{0}\right)}=$ $\mathcal{O}_{X \cap D_{+}\left(x_{0}\right)} \cdot \eta$, where

$$
\begin{aligned}
\eta & =( \pm) \frac{d \xi_{2} \wedge \cdots \wedge d \xi_{n}}{\frac{\partial}{\partial \xi_{1}}\left(\nu^{p}-f^{\prime}\right)}=( \pm) \frac{d \xi_{1} \wedge d \xi_{3} \wedge \cdots \wedge d \xi_{n}}{\frac{\partial}{\partial \xi_{2}}\left(\nu^{p}-f^{\prime}\right)} \\
& =\cdots=( \pm) \frac{d \xi_{1} \wedge \cdots \wedge d \xi_{n-1}}{\frac{\partial}{\partial \xi_{n}}\left(\nu^{p}-f^{\prime}\right)}
\end{aligned}
$$

is a $(n-1)$-form on $X$.

Let $l$ be a sufficiently divisible positive integer so that $\mathcal{M}^{[l]}$ is an invertible sheaf on $X$, where $\mathcal{M}^{[l]}$ is the double dual of $\mathcal{M}^{\otimes l}$. Then, there are integers $\varepsilon_{i}^{\prime}$ such that

$$
\mathcal{L}^{\otimes l}=\varphi^{*} \mathcal{M}^{[l]} \otimes \mathcal{O}_{Y}\left(-\sum_{i} \varepsilon_{i}^{\prime} E_{i}\right) .
$$

Put $\varepsilon_{i}=\varepsilon_{i}^{\prime} / l$. The rational number $\varepsilon_{i}$ does not depend on the choice of $l$.

Definition 4.7. For each $I \in \mathcal{I}$, let

$$
Z_{I}:=\left(\bigcap_{i \in\{0, \ldots, n+1\} \backslash I}\left(x_{i}=0\right)\right) \cap\left(\bigcap_{i \in I}\left(x_{i} \neq 0\right)\right) \cap X_{\mathrm{qs}}
$$

be a locally closed subset of $X$. We call $Z_{I}$ a singular stratum of $X$. 
By Lemma 3.6 and the definition of $\mathcal{I}$, we see that

$$
\operatorname{Sing}(X) \cap\left(x_{0}=0\right)=\bigcup_{I \in \mathcal{I}} Z_{I},
$$

where the union is disjoint.

To conclude that the line bundle $\mathcal{L}$ is big, we need to lift global sections of $\mathcal{M}^{[l]}$ to those of $\mathcal{L}^{\otimes l}$. In other words, we need to bound the rational number $\varepsilon_{i}$ from above. The proof of the following lemma will be postponed until the next section.

Lemma 4.8. Let $E_{i}$ be an exceptional divisor of $\varphi: Y \rightarrow X$ whose center is the closure $\bar{Z}_{I}$ of a stratum $Z_{I}$ for some $I \subset\{1, \ldots, n+1\}$. Then, we have $A>r_{I} \varepsilon_{i}$.

Lemma 4.9. $\mathcal{L}$ is a big line bundle on $Y$.

Proof. Put $a_{\max }=\max \left\{a_{1}, \ldots, a_{n}\right\}$. By Lemma 4.8, we have $l \varepsilon_{i} \leq$ $A l / r_{I}-a_{\max }$ for all sufficiently large and divisible $l$. We see that

$$
\begin{aligned}
\varphi_{*} \mathcal{L}^{\otimes l} & =\mathcal{M}^{[l]} \otimes \varphi_{*} \mathcal{O}_{Y}\left(-\sum_{i} l \varepsilon_{i} E_{i}\right) \\
& \supset \mathcal{M}^{[l]} \otimes \varphi_{*} \mathcal{O}_{Y}\left(-\sum_{\varepsilon_{i}>0}\left(A l / r_{I}-a_{\max }\right) E_{i}\right) .
\end{aligned}
$$

Consider the global sections $x_{0}^{A l}, x_{0}^{A l-a_{1}} x_{1}, \ldots, x_{0}^{A l-a_{n}} x_{n}$ of $\mathcal{M}^{[l]} \cong \mathcal{O}_{X}(A l)$. Let $U$ be a sufficiently small open subset of $X$ such that $U \cap Z_{I} \neq \emptyset$. Then $\left.x_{0}^{r_{I}}\right|_{U} \in \mathcal{O}_{U}$ and it vanish along $\bar{Z}_{I}$. Hence, for each $i$, the section $x_{0}^{A l-a_{i}} x_{i}=\left(x_{0}^{r_{I}}\right)^{A l / r_{I}-a_{i}} x_{0}^{\left(r_{I}-1\right) a_{i}} x_{i}$ vanishes along each singular stratum $\bar{Z}_{I}$ with multiplicity at least $A l / r_{I}-a_{\max }$ and thus lifts to a global section of $\mathcal{L}^{\otimes l}$.

The global sections $x_{0}^{A l}, \ldots, x_{0}^{A l-a_{n}} x_{n}$ define a dominant map $X \rightarrow \mathbb{P}^{n}$. Therefore, $\mathcal{L}$ is big.

\section{§5. Local models of $X_{\mathrm{qs}}$}

This section is devoted to prove Lemma 4.8 .

\subsection{Preparation from toric geometry}

Let us fix some basic notations on toric varieties. For details, we refer the reader to [9]. Let $\mathrm{N}$ be a lattice of $\operatorname{rank} n, \mathrm{M}=\operatorname{Hom}(\mathrm{N}, \mathbb{Z})$ its dual, $\sigma$ a strictly convex rational polyhedral cone in $\mathrm{N}_{\mathbb{R}}=\mathrm{N} \otimes_{\mathbb{Z}} \mathbb{R}$ and $k$ an algebraically closed field. Then, the scheme $S:=k\left[\sigma^{\vee} \cap \mathrm{M}\right]$ is an affine 
normal variety which we call the affine toric variety defined by $(\mathrm{N}, \sigma)$ over $k$. Such an variety $S$ contains the $n$-dimensional torus $T:=\operatorname{Spec} k[\mathrm{M}]$. We can identify $\mathrm{N}$ (resp. M) with the group of homomorphisms of algebraic groups from $\mathbb{G}_{\mathrm{m}}$ to $T$ (resp. from $T$ to $\mathbb{G}_{\mathrm{m}}$ ). If $\alpha \in \mathrm{M}$ then we denote by $\chi^{\alpha}$ the corresponding element of $\Gamma\left(T, \mathcal{O}_{T}\right)$ and if $\beta \in \mathrm{N}$ then we denote by $\lambda_{\beta}: \mathbb{G}_{\mathrm{m}} \rightarrow T$ the corresponding homomorphism. Let $\tau$ be a face of $\sigma$ and $\beta$ be any point of $\operatorname{Int}(\tau) \cap \mathrm{N}$. Then, the limit $\lim _{t \rightarrow 0} \lambda_{\beta}(t)$ exists in $S$ and is uniquely determined by $\tau$, that is, if $\beta_{1}, \beta_{2} \in \mathrm{N}$ then we have $\lim _{t \rightarrow 0} \lambda_{\beta_{1}}(t)=$ $\lim _{t \rightarrow 0} \lambda_{\beta_{2}}(t)$ if and only if $\beta_{1}$ and $\beta_{2}$ lie in the interior of some face of $\sigma$. We call $\lim _{t \rightarrow 0} \lambda_{\beta}(t)$ the distinguished point which corresponds to $\tau$.

Definition 5.1. For a positive integer $r$ and integers $c_{1}, \ldots, c_{n}$, let

$$
\mathrm{N}=\mathrm{N}\left(r ; c_{1}, \ldots, c_{n}\right):=\mathbb{Z} \cdot e_{1}+\cdots+\mathbb{Z} \cdot e_{n}+\mathbb{Z} \cdot(1 / r)\left(c_{1} e_{1}+\cdots+c_{n} e_{n}\right)
$$

be the lattice of rank $n$ and $\mathrm{M}=\mathrm{M}\left(r ; c_{1}, \ldots, c_{n}\right)$ be the dual lattice of $\mathrm{N}$. For a subset $\left\{i_{1}, \ldots, i_{k}\right\}$ of $\{1, \ldots, n\}$, let

$$
\sigma=\sigma\left(i_{1}, \ldots, i_{k}\right):=\mathbb{R}_{\geq 0} \cdot e_{i_{1}}+\cdots+\mathbb{R}_{\geq 0} \cdot e_{i_{k}}
$$

be the strictly convex rational polyhedral cone in $N_{\mathbb{R}}$.

For each $i=1, \ldots, n$, put

$$
\delta_{i}=\operatorname{gcd}\left\{r, c_{1}, \ldots, \hat{c}_{i}, \ldots, c_{n}\right\} / \operatorname{gcd}\left\{r, c_{1}, \ldots, c_{n}\right\} \in \mathbb{Z}
$$

For a lattice point $\alpha \in \mathrm{M}$, let $\alpha^{(1)}, \ldots, \alpha^{(n)}$ be the integers such that $\alpha=$ $\alpha^{(1)} e_{1}^{*}+\cdots+\alpha^{(n)} e_{n}^{*}$, where $\left\{e_{1}^{*}, \ldots, e_{n}^{*}\right\}$ is the dual basis of $\left\{e_{1}, \ldots, e_{n}\right\}$. Let $\mathbb{k}$ be an algebraically closed field of characteristic $p$. We denote by $V$ the $\mathbb{k}$-vector space $M \otimes_{\mathbb{Z}} \mathbb{k}$.

Definition 5.2. Let $\mathrm{N}$ be a lattice, $\mathrm{M}$ a dual lattice of $\mathrm{N}, \sigma$ a strictly convex rational polyhedral cone in $\mathrm{N}_{\mathbb{R}}$ and $S$ the affine toric variety defined by $(\mathrm{N}, \sigma)$. For a lattice points $\alpha_{1}, \ldots, \alpha_{q} \in \mathrm{M}$, we denote by $\omega\left(\alpha_{1}, \ldots, \alpha_{q}\right)$ the rational $q$-form

$$
\omega\left(\alpha_{1}, \ldots, \alpha_{q}\right)=\frac{d \chi^{\alpha_{1}} \wedge \cdots \wedge d \chi^{\alpha_{q}}}{\chi^{\alpha_{1}+\cdots+\alpha_{q}}}
$$

on $S$. 
LEMMA 5.3. Let $p$ be a prime number, $r$ a positive integer and $c_{1}, \ldots, c_{n}$ integers. Let $S$ be the affine toric variety defined by

$$
(\mathrm{N}, \sigma)=\left(\mathrm{N}\left(r ; c_{1}, \ldots, c_{n}\right), \sigma(1, \ldots, k)\right)
$$

over $\mathbb{k}$. Suppose that we are given lattice points $\alpha_{1}, \ldots, \alpha_{q} \in \mathrm{M}=\operatorname{Hom}(\mathrm{N}, \mathbb{Z})$ such that $\alpha_{1} \wedge \cdots \wedge \alpha_{q} \neq 0$ in $\wedge^{q} V$ and put $\omega=\omega\left(\alpha_{1}, \ldots, \alpha_{q}\right)$. Let $l$ be $a$ positive integer and $\alpha \in \mathrm{M}$. Then, we have

$$
\chi^{\alpha} \omega^{\otimes l} \in H^{0}\left(S,\left(\Omega_{S}^{q}\right)^{[l]}\right)
$$

if and only if the following holds for every $i=1, \ldots, k$ :

$$
\alpha^{(i)} \geq \begin{cases}0 & \text { if } \alpha_{j}^{(i)} / \delta_{i} \equiv 0(\bmod p) \text { for every } j=1, \ldots, q \\ l \delta_{i} & \text { otherwise }\end{cases}
$$

Proof. For each $i=1, \ldots, k$, let $\sigma_{i}=\mathbb{R}_{\geq 0} \cdot e_{i}$ be the 1-dimensional face of $\sigma$ and let

$$
S_{i}=\operatorname{Spec} k\left[\sigma_{i} \vee \cap M\right]
$$

be the open subvariety of $S$. The variety $S_{i}$ is nonsingular and the codimension of $S \backslash \bigcup_{i=1}^{k} S_{i}$ in $S$ is 2 . Thus, $\left.\chi^{\alpha} \omega^{\otimes l} \in H^{0}\left(S,\left(\Omega_{S}^{q}\right)^{[l]}\right)\right)$ if and only if $\chi^{\alpha} \omega^{\otimes l}$ is holomorphic on $S_{i}$ for every $i=1, \ldots, k$.

Choose and fix any $i \in\{1, \ldots, k\}$. Put $m_{j}=\alpha_{j}^{(i)} / \delta_{i}$ and $m=\alpha^{(i)} / \delta_{i} \in$ $\mathbb{Z}$. We can take affine coordinates $z_{1}, \ldots, z_{n}$ so that we have

$$
S_{i} \cong \operatorname{Spec} \mathbb{k}\left[z_{1}, \ldots, z_{n}, z_{1}^{-1}, \ldots, \widehat{z_{i}^{-1}}, \ldots, z_{n}^{-1}\right] .
$$

Moreover, under the isomorphism, we have $\chi^{\alpha}=z_{i}^{m} h$ and $\chi^{\alpha_{j}}=z_{i}^{m_{j}} h_{j}$, where $h, h_{j}$ are functions of $z_{1}, \ldots, \hat{z}_{i}, \ldots, z_{n}$. Therefore, we have

$$
\left.\chi^{\alpha} \omega^{\otimes l}\right|_{S_{i}}=z_{i}^{m} h\left(\frac{d\left(z_{i}^{m_{1}} h_{1}\right) \wedge \cdots \wedge d\left(z_{i}^{m_{q}} h_{q}\right)}{z_{i}^{m_{1}+\cdots+m_{q}} h_{1} \cdots h_{q}}\right)^{\otimes l} .
$$

Since $h$ and $h_{j}$ are unit element in $\mathcal{O}_{U_{i}}$, by a direct computation, the lemma is proved.

For a prime number $p$ and a nonnegative integer $c$, we define $\operatorname{mult}_{p}(c):=$ $\max \left\{m \mid c \in p^{m} \mathbb{Z}\right\}$ if $c \neq 0$ and $\operatorname{mult}_{p}(c):=\infty$ if $c=0$. If $c=0$ then we define $p^{-\operatorname{mult}_{p}(c)}:=0$. 
COROLlary 5.4. Let $p$ be a prime number, $r$ a positive integer and $c_{1}, \ldots, c_{n}$ integers such that $0 \leq c_{i}<r$ for $i=1, \ldots, n$. Assume that

$$
\operatorname{mult}_{p}\left(c_{1}\right) \leq \operatorname{mult}_{p}\left(c_{2}\right) \leq \cdots \leq \operatorname{mult}_{p}\left(c_{n-1}\right) .
$$

Let $S$ be the affine toric variety defined by

$$
(\mathrm{N}, \sigma)=\left(\mathrm{N}\left(r ; c_{1}, \ldots, c_{n}\right), \sigma(1, \ldots, k)\right)
$$

over $\mathbb{k}$ and

$$
\omega=\omega\left(r^{\prime} e_{1}^{*}, c_{1}^{\prime} e_{2}^{*}-c_{2}^{\prime} e_{1}^{*}, \ldots, c_{1}^{\prime} e_{n-1}^{*}-c_{n-1}^{\prime} e_{1}^{*}\right)
$$

be the rational $(n-1)$-form on $S$, where $r^{\prime}=r p^{-\min \left\{\operatorname{mult}_{p}(r), \operatorname{mult}_{p}\left(c_{1}\right)\right\}}$ and $c_{i}^{\prime}=c_{i} p^{- \text {mult }_{p}\left(c_{1}\right)}$. Suppose we are given a positive integer $l$ which is divisible by $r$. Then, we have

$$
\mathbb{k}(S) \cdot \omega^{\otimes l} \cap\left(\Omega_{S}^{n-1}\right)^{[l]} \subset \mathcal{O}_{S} \cdot \chi^{l\left(e_{1}^{*}+\cdots+e_{k^{\prime}}^{*}\right)} \omega^{\otimes l},
$$

where $k^{\prime}=\min \{k, n-1\}$.

Proof. It can be checked that

$$
\alpha_{1}:=r^{\prime} e_{1}^{*}, \alpha_{2}:=c_{1}^{\prime} e_{2}^{*}-c_{2}^{\prime} e_{1}^{*}, \ldots, \alpha_{n-1}:=c_{1}^{\prime} e_{n-1}^{*}-c_{n-1}^{\prime} e_{1}^{*}
$$

form a basis of $V$. Set $s=\operatorname{mult}_{p}(r)$ and $t_{i}=\operatorname{mult}_{p}\left(c_{i}\right)$. By Lemma 5.3, it suffices to show that the integer $\alpha_{i}^{(1)} / \delta_{1}$ is not divisible by $p$ for some $i=1, \ldots, n-1$, since $p \nmid \alpha_{i}^{(i)}=c_{1}^{\prime}$ for $i=2, \ldots, n-1$. If $t_{1}=t_{2}$ then $\alpha_{2}^{(1)}=c_{2}^{\prime}$ is not divisible by $p$. If $s \leq t_{1}$ then $\alpha_{1}^{(1)}=r^{\prime}=r / p^{s}$ is not divisible by $p$. We assume that $t_{1}<t_{2}$ and $t_{1}<s$. In this case $\operatorname{mult}_{p}\left(\alpha_{1}^{(1)}\right)=s-t_{1}$ and $\operatorname{mult}_{p}\left(\alpha_{2}^{(1)}\right)=t_{2}-t_{1}$. By the definition of $\delta_{1}$, we have $\operatorname{mult}_{p}\left(\delta_{1}\right)=$ $\min \left\{s, t_{2}\right\}-t_{1}$. If $s \leq t_{2}$ then we have

$$
\operatorname{mult}_{p}\left(\alpha_{1}^{(1)} / \delta_{1}\right)=\operatorname{mult}_{p}\left(\alpha_{1}^{(1)}\right)-\operatorname{mult}_{p}\left(\delta_{1}\right) \leq\left(s-t_{1}\right)-\left(s-t_{1}\right)=0 .
$$

If $s \geq t_{2}$ then we have

$$
\operatorname{mult}_{p}\left(\alpha_{2}^{(1)} / \delta_{1}\right)=\left(t_{2}-t_{1}\right)-\left(t_{2}-t_{1}\right)=0 .
$$

This completes the proof. 
Corollary 5.5. Let $p$ be a prime number, $r$ a positive integer and $c_{1}, \ldots, c_{n}$ nonnegative integers. Assume that $c_{1}$ and $c_{2}$ are not divisible by $p$. Let $S$ be the affine toric variety defined by

$$
(\mathrm{N}, \sigma)=\left(\mathrm{N}\left(r ; c_{1}, \ldots, c_{n}\right), \sigma(1, \ldots, n)\right)
$$

over $\mathbb{k}$ and

$$
\omega=\omega\left(c_{1} e_{2}^{*}-c_{2} e_{1}^{*}, c_{1} e_{3}^{*}-c_{3} e_{1}^{*}, \ldots, c_{1} e_{n}^{*}-c_{n} e_{1}^{*}\right)
$$

be the rational $(n-1)$-form on $S$. Suppose we are given a positive integer $l$ which is divisible by $r$. Then, we have

$$
\mathbb{k}(S) \cdot \omega^{\otimes l} \cap\left(\Omega_{S}^{n-1}\right)^{[l]} \subset \mathcal{O}_{S} \cdot \chi^{l\left(e_{1}^{*}+\cdots+e_{n}^{*}\right)} \omega^{\otimes l}
$$

Proof. It can be checked that

$$
\alpha_{1}:=r e_{1}^{*}, \alpha_{2}:=c_{1} e_{2}^{*}-c_{2} e_{1}^{*}, \ldots, \alpha_{n}:=c_{1} e_{n}^{*}-c_{n} e_{1}^{*}
$$

form a basis of $V=\mathrm{M} \otimes_{\mathbb{Z}} \mathbb{k}$. We have $\operatorname{mult}_{p}\left(\alpha_{i}^{(i)}\right)=\operatorname{mult}_{p}\left(c_{1}\right)=0$ for $i=2, \ldots, n$ and $\operatorname{mult}_{p}\left(\alpha_{2}^{(1)}\right)=\operatorname{mult}_{p}\left(c_{2}\right)=0$. Thus, the Lemma follows from Lemma 5.3.

\subsection{Description of local models}

Throughout this subsection, we assume Assumption 4.1.

Definition 5.6. For $I \in \mathcal{I}_{\text {sat }}$ with $I \neq\{n+1\}$, put $m=\left|I^{\#}\right|, \tilde{n}=$ $n-(m+1)$ and let $c_{1}, \ldots, c_{m}$ be the positive integers such that $\left\{c_{1}, \ldots, c_{m}\right\}=$ $\left\{\left[a_{i}\right]^{I} \mid i \in I^{\#}\right\}, \operatorname{mult}_{p}\left(c_{1}\right) \leq \cdots \leq \operatorname{mult}_{p}\left(c_{m}\right)$ and if $\operatorname{mult}_{p}\left(c_{i}\right)=\operatorname{mult}_{p}\left(c_{j}\right)$ then $c_{i} \leq c_{j}$. Then, let

$$
\mathrm{N}_{I}:=\mathrm{N}\left(r_{I} ; c_{1}, \ldots, c_{m}, 0, \ldots, 0, b\right)
$$

be the lattice of rank $n, \mathrm{M}_{I}$ its dual and let

$$
\sigma_{I}:= \begin{cases}\mathbb{R}_{\geq 0} \cdot e_{1}+\cdots+\mathbb{R}_{\geq 0} \cdot e_{m}+\mathbb{R}_{\geq 0} \cdot e_{n}, & \text { if } n+1 \notin I, \\ \mathbb{R}_{\geq 0} \cdot e_{1}+\cdots+\mathbb{R}_{\geq 0} \cdot e_{m}, & \text { if } n+1 \in I,\end{cases}
$$

be the cone in $\left(\mathrm{N}_{I}\right)_{\mathbb{R}}$. We denote by $S_{I}$ the affine toric variety defined by $\left(\mathrm{N}_{I}, \sigma_{I}\right)$ over $\mathbb{k}$ and by $s_{I}$ the distinguished point of $S_{I}$ which corresponds to the cone $\sigma_{I}$ in $\left(\mathrm{N}_{I}\right)_{\mathbb{R}}$. Let $\alpha_{I}:=e_{1}^{*}+\cdots+e_{m}^{*}$ be the point of $\sigma_{I}^{\vee}$ and let

$$
\omega_{I}:=d\left(c_{1}^{\prime} e_{1}^{*}, c_{1}^{\prime} e_{2}^{*}-c_{2}^{\prime} e_{1}^{*}, \ldots, c_{1}^{\prime} e_{m}^{*}-c_{m}^{\prime} e_{1}^{*}, e_{m+1}^{*}, \ldots, e_{n-1}^{*}\right)
$$


be the rational $(n-1)$-form on $S_{I}$, where $c_{i}^{\prime}=c_{i} p^{-\operatorname{mult}_{p}\left(c_{i}\right)}$.

When $I=\{n+1\}$, after renumbering the indices of $a_{1}, \ldots, a_{n}$, we assume that Condition (2.3.2) holds for $j=1$ and $0=\operatorname{mult}_{p}\left(a_{1}\right)=\operatorname{mult}_{p}\left(a_{2}\right)$ $\leq \operatorname{mult}_{p}\left(a_{3}\right) \leq \cdots \leq \operatorname{mult}_{p}\left(a_{n}\right)$. We define

$$
\left(\mathrm{N}_{I}, \sigma_{I}\right):=\left(\mathrm{N}\left(b ; a_{1}, \ldots, a_{n}\right), \mathbb{R}_{\geq 0} \cdot e_{1}+\cdots+\mathbb{R}_{\geq 0} \cdot e_{n}\right)
$$

and let $\mathrm{M}_{I}$ be the dual lattice of $\mathrm{N}_{I}$. We denote by $S_{I}$ the affine toric variety defined by $\left(\mathrm{N}_{I}, \sigma_{I}\right)$ over $\mathbb{k}$ and by $s_{I}$ the distinguished point of $S_{I}$ which corresponds to the cone $\sigma_{I}$ in $\left(\mathrm{N}_{I}\right)_{\mathbb{R}}$. Let $\alpha_{I}:=e_{1}^{*}+\cdots+e_{n}^{*}$ be the point of $\sigma_{I}^{\vee}$ and let

$$
\omega_{I}:=d\left(a_{1} e_{2}^{*}-a_{2} e_{1}^{*}, a_{1} e_{3}^{*}-a_{3} e_{1}^{*}, \ldots, a_{1} e_{n}^{*}-a_{n} e_{1}^{*}\right)
$$

be the rational $(n-1)$-form on $S_{I}$.

Throughout this subsection, we choose and fix a sufficiently divisible positive integer $l$.

LEMMA 5.7. Let $I$ be a subset of $\{1, \ldots, n+1\}$ such that $I$ is saturated or $I=\{n+1\}$ and let $x \in Z_{I}$ be any closed point. Then the following assertions hold.

(1) $\left(S_{I}, s_{I}\right)$ is a local model of $\left(X_{\mathrm{qS}}, x\right)$.

(2) We have $\mathbb{k}\left(S_{I}\right) \cdot \omega_{I}^{\otimes l} \cap\left(\Omega_{S_{I}}^{n-1}\right)^{[l]} \subset \mathcal{O}_{S_{I}} \cdot \chi^{l \alpha_{I}} \omega_{I}^{\otimes l}$.

(3) There is a rational $(n-1)$-form $\omega_{x}$ on $X$ such that $\mathcal{M}_{x}^{[l]} \subset \mathbb{k}(X) \cdot \omega_{x}^{\otimes l}$ and $\omega_{x}$ is identified with $\omega_{I}$ by the isomorphism $\hat{\mathcal{O}}_{X, x} \cong \hat{\mathcal{O}}_{S_{I}, s_{I}}$.

(4) There is a function $h_{x} \in \mathcal{O}_{X, x}$ such that it can be identified with $\chi^{\text {lo } \alpha_{I}}$ by the isomorphism $\hat{\mathcal{O}}_{X, x} \cong \hat{\mathcal{O}}_{S_{I}, s_{I}}$.

(5) Let $\varphi_{I}: S^{\prime} \rightarrow S_{I}$ be a toric resolution obtained by subdividing the cone $\sigma_{I}$. Then, for any exceptional divisor $E^{\prime}$ of $\varphi_{I}$, the order of the pole of $\varphi_{I}^{*}\left(\chi^{l \alpha_{I}} \omega_{I}^{\otimes l}\right)$ along $E^{\prime}$ is at most $A l / r_{I}-1$.

Proof. Let $x \in Z_{I}$ be a point and $|I|=k$. For each $I \subset\{1, \ldots, n+1\}$, we associate an affine variety

$C_{\mathbb{P}, I}:=\operatorname{Spec} \mathbb{k}\left[\left\{\xi_{i} \mid i \in\{0, \ldots, n+1\} \backslash I\right\}\right] \times \operatorname{Spec} \mathbb{k}\left[u_{1}, u_{1}^{-1}, \ldots, u_{k-1}, u_{k-1}^{-1}\right]$, 
where $\mathbb{P}=\mathbb{P}\left(1, a_{1}, \ldots, a_{n}, b\right)$. The group scheme $\boldsymbol{\mu}_{r_{I}}$ acts on $U_{I}$ by $\xi_{i} \mapsto$ $\xi_{i} \otimes \bar{t}^{a_{1}}, u_{j} \mapsto u_{j}$ and gives the quotient $\left(C_{\mathbb{P}, I} / \boldsymbol{\mu}_{r_{I}}\right) \cong \mathrm{D}_{+}\left(x_{I}\right)$ (cf. DefinitionLemma 3.2). There is a natural morphism

$$
\tau_{I}: C_{\mathbb{P}, I} \longrightarrow\left(C_{\mathbb{P}, I} / \boldsymbol{\mu}_{r_{I}}\right) \cong \mathrm{D}_{+}\left(x_{I}\right)
$$

The locally closed subset $Z_{I}$ is exactly the closed subset $X \cap\left(\bigcap_{i \notin I}\left(\xi_{i}=0\right)\right)$ of $X \cap \mathrm{D}_{+}\left(x_{I}\right)$. Let $f_{I}, g_{I}$ be the functions of $\left\{\xi_{i} \mid i \notin I\right\}$ and $u_{1}, \ldots, u_{k-1}$ which correspond to $f, y^{p} x_{0}$ respectively. Then, we see that $C_{X, I}=\left(g_{I}-f_{I}=\right.$ 0) $\subset C_{\mathbb{P}, I}$. The group scheme $\boldsymbol{\mu}_{r_{I}}$ acts on $C_{X, I}$ and gives the quotient $C_{X, I} / \boldsymbol{\mu}_{r_{I}}=X \cap \mathrm{D}_{+}\left(x_{I}\right)$. Since $X_{\mathrm{qs}}$ is quasi smooth, there is some $i_{0} \notin I$ (or $j \in\{1, \ldots, k-1\}$ ) such that we can choose $\left\{\xi_{i} \mid i \notin I, i \neq i_{0}\right\}$ and $u_{1}, \ldots, u_{k-1}$ (or $\left\{\xi_{i} \mid i \notin I\right\}$ and $u_{1}, \ldots, \hat{u}_{j}, \ldots, u_{k-1}$ ) as local coordinates of $C_{X, I}$ at the point $\tau_{I}^{-1}(z)$. If $n+1 \notin I$, then we write $\nu$ instead of $\xi_{n+1}$ in the following. In this case, the coordinate $\nu$ corresponds to the homogeneous coordinate $y$ and we have $g_{I}=\nu^{p} \xi_{0}$. By this observation, we can describe a local model of $X_{\mathrm{qs}}$ at $z \in Z_{I}$. To go further, we divide the proof of (1)-(4) into four cases.

Case 1: $I=\{k+1, \ldots, n\}$ is saturated and $r_{I} \mid d$.

In this case, we can write $f_{I}=f^{\prime}+f^{\prime \prime}$, where $f^{\prime}=f^{\prime}\left(u_{1}, \ldots, u_{n-k-1}\right)$ and $f^{\prime \prime} \in\left(\xi_{0}, \ldots, \xi_{k}\right)^{2}$ since $I$ is saturated and $r_{I} \mid d$. Hence, $\left(\partial\left(g_{I}-\right.\right.$ $\left.\left.f_{I}\right) / \partial \xi_{i}\right)(x)=0$ for $i=0, \ldots, k$ and, by the smoothness of $C_{X, I}$, we may assume that $\xi_{0}, \ldots, \xi_{k}, \nu, u_{1}, \ldots, u_{n-k-2}$ form a local coordinates of $C_{X, I}$ at $\tau_{I}^{-1}(x)$. The action of $\boldsymbol{\mu}_{r_{I}}$ on $C_{X, I}$ is given by $\xi_{i} \mapsto \xi_{i} \otimes \bar{t}^{a_{i}}, \nu \mapsto \nu \otimes \bar{t}^{b}$ and $u_{i} \mapsto u_{i}$. Thus, we see that $\left(S_{I}, s_{I}\right)$ is a local model model of $X_{\mathrm{qs}}$ at $x$. We may assume that $\xi_{i}\left(\right.$ resp. $\left.u_{j}\right)$ corresponds to $\chi^{e_{i}^{*}}\left(\right.$ resp. $\left.\chi^{\tilde{e}_{j}^{*}}\right)$ for $i=0, \ldots, k$ (resp. $j=1, \ldots, n-k-2$ ) after passing to the completion.

Let

$$
\omega_{x}=\frac{d \xi_{0}^{r_{I}} \wedge d\left(\xi_{1} / \xi_{0}^{a_{1}}\right) \wedge \cdots \wedge d\left(\xi_{k} / \xi_{0}^{a_{k}}\right) \wedge d u_{1} \wedge \cdots \wedge d u_{n-k-2}}{\xi_{0}^{r_{I}}\left(\xi_{1} / \xi_{0}^{a_{1}}\right) \cdots\left(\xi_{k} / \xi_{0}^{a_{k}}\right) u_{1} \cdots u_{n-k-2}}
$$

be the rational $(n-1)$-form on $X$. We see that, after passing to the completion, $\omega_{x}$ can be identified with $\omega_{I}$. Let $\xi_{1}^{\prime}, \ldots, \xi_{n}^{\prime}, \nu^{\prime}$ be the natural affine coordinates of $\mathrm{D}_{+}\left(x_{0}\right)$ defined by $\xi_{i}^{\prime}=x_{i} / x_{0}^{a_{i}}$ for $i=1, \ldots, n$ and $\nu^{\prime}=y / x_{0}^{b}$. If we restrict $\omega_{x}$ on $X \cap \mathrm{D}_{+}\left(x_{I}\right) \cap \mathrm{D}_{+}\left(x_{0}\right)$, it can be written as

$$
\omega_{x}=\frac{d G_{1} \wedge \cdots \wedge d G_{n-1}}{G_{1} \cdots G_{n-1}}
$$


where $G_{i}$ is a monomial of $\xi_{1}^{\prime}, \ldots, \xi_{n}^{\prime}$ for every $i$. Then, by Remark 4.6, there is a rational function $\Psi$ on $X$ such that $\omega_{x}=\Psi \eta$. Therefore, we see that

$$
\mathcal{M}_{x}^{[l]} \subset \mathbb{k}(X) \cdot \eta^{\otimes l}=\mathbb{k}(X) \cdot \omega_{x}^{\otimes l} .
$$

By Corollary 5.4, we have

$$
\mathcal{O}_{S_{I}} \cdot \omega_{I}^{\otimes l} \cap\left(\Omega_{S_{I}}^{n-1}\right)^{[l]} \subset \mathcal{O}_{S_{I}} \cdot \chi^{l \alpha_{I}} \omega_{I}^{\otimes l},
$$

where, in this case, $\alpha_{I}=e_{1}^{*}+\cdots+e_{k+1}^{*}$. Put $h_{x}=\xi_{0}^{l} \cdots \xi_{k}^{l}$. Then, the function $h_{x} \in \mathcal{O}_{X, x}$ is identified with $\chi^{l \alpha_{I}}$ after passing to the completion.

Case 2: $I=\{k+1, \ldots, n\}$ is saturated and $r_{I} \nmid d$.

In this case, we can write $f_{I}=f_{0}^{\prime} \xi_{0}+\cdots+f_{k}^{\prime} \xi_{k}+f^{\prime \prime}$, where $f_{i}^{\prime}=$ $f_{i}^{\prime}\left(u_{1}, \ldots, u_{n-k-1}\right)$ and $f^{\prime \prime} \in\left(\xi_{0}, \ldots, \xi_{k}\right)^{2}$ since $I$ is saturated and $r_{I} \nmid d$. Hence, we have $\left(\partial\left(g_{I}-f_{I}\right) / \partial u_{i}\right)(z)=0$ and $\left(\partial\left(g_{I}-f_{I}\right) / \partial \xi_{i}\right)(z)=f_{i}^{\prime}(z)$ for $z \in Z_{I}$. By the smoothness of $C_{I}$, there is some $j \in\{0, \ldots, k\}$ such that $f_{j}^{\prime}(z) \neq 0$. Such a $j$ necessarily satisfies $d-a_{j} \equiv 0\left(\bmod r_{I}\right)$. For simplicity of the proof, we assume that $j=0$. Note that the description of $\omega_{x}$ below depends on the choice of $j$ and we need to consider the case $j \in\{1, \ldots, k\}$ for a complete proof. But, in that case, the description of $\omega_{x}$ is similar and, moreover, rather easy compared to the case $j=0$. Hence, we concentrate on the case $j=0$. We see that $\xi_{1}, \ldots, \xi_{k}, u_{1}, \ldots, u_{n-k-1}$ form a local coordinates of $C_{I}$ at $\tau_{I}^{-1}(z)$. After renumbering the indices of $a_{1}, \ldots, a_{k}$, we may assume that $c_{i}=\left[a_{i}\right]^{I}$ for $i=1, \ldots, k$ (See Definition 5.6 for the definition of $\left.c_{i}\right)$. Thus, we see that $\left(S_{I}, s_{I}\right)$ is a local model of $X_{\mathrm{qS}}$ at $x$ and, after passing to the completion, $\xi_{i}$ (resp. $u_{j}$ ) is identified with $\chi^{e_{i}^{*}}$ (resp. $\chi^{\tilde{e}_{j}^{*}}$ ) for $i=1, \ldots, k$ (resp. $j=1, \ldots, n-k-1$ ). Let

$$
\omega_{x}=\frac{d \xi_{1}^{r_{I}^{\prime}} \wedge d\left(\xi_{2}^{c_{1}^{\prime}} / \xi_{1}^{c_{2}^{\prime}}\right) \wedge \cdots \wedge d\left(\xi_{k}^{c_{1}^{\prime}} / \xi_{1}^{c_{k}^{\prime}}\right) \wedge d u_{1} \wedge \cdots \wedge d u_{n-k-1}}{\xi_{1}^{r_{I}^{\prime}}\left(\xi_{2}^{c_{1}^{\prime}} / \xi_{1}^{c_{2}^{\prime}}\right) \cdots\left(\xi_{k}^{c_{1}^{\prime}} / \xi_{1}^{c_{k}^{\prime}}\right) u_{1} \cdots u_{n-k-1}}
$$

be the rational $(n-1)$-form on $X$, where $c_{i}^{\prime}=c_{i} p^{-\operatorname{mult}_{p}\left(c_{1}\right)}$ and $r_{I}^{\prime}=$ $r_{I} p^{-\min \left\{\operatorname{mult}_{p}\left(r_{I}\right), \operatorname{mult}_{p}\left(c_{1}\right)\right\}}$. We see that, after passing to the completion, $\omega_{x}$ can be identified with $\omega_{I}$ and, as in the case 1 , we have $\mathcal{M}_{z}^{[l]} \subset \mathcal{O}_{X, z} \cdot \omega_{x}^{\otimes l}$. By Corollary 5.3, we have

$$
\mathbb{k}\left(S_{I}\right) \cdot \omega_{I}^{\otimes l} \cap\left(\Omega_{S_{I}}^{n-1}\right)^{[l]} \subset \mathcal{O}_{S} \cdot \chi^{l \alpha_{I}} \omega_{I}^{\otimes l},
$$

where, in this case, $\alpha_{I}=e_{1}^{*}+\cdots+e_{k}^{*}$. Put $h_{x}=\xi_{1}^{l} \cdots \xi_{k}^{l}$. Then the function $h_{x}$ is identified with $\chi^{l \alpha_{I}}$ after passing to the completion. 
Case 3: $I=\{k+1, \ldots, n+1\}$ is saturated.

Since $r_{I} \nmid d$ and $I$ is saturated, we can write $f_{I}=f_{0}^{\prime} \xi_{0}+\cdots+f_{k}^{\prime} \xi_{k}+f^{\prime \prime}$, where $f^{\prime}=f^{\prime}\left(u_{1}, \ldots, u_{n-k-1}\right)$ and $" \in\left(\xi_{0}, \ldots, \xi_{k}\right)^{2}$. As in the case 2, we have $\left(\partial\left(g_{I}-f_{I}\right) / \partial \xi_{j}\right)(z)=f_{j}^{\prime}(z) \neq 0$ for some $j \in\{0, \ldots, k\}$ such that $a_{j} \equiv d \equiv 1\left(\bmod r_{I}\right)$. For simplicity of the proof, we assume that $j=0$. Therefore, we may assume that $\xi_{1}, \ldots, \xi_{k}, u_{1}, \ldots, u_{n-k}$ form a local coordinates of $C_{I}$ at $\tau_{I}^{-1}(z)$. After renumbering the indices of $a_{1}, \ldots, a_{k}$, we may assume that $c_{i}=\left[a_{i}\right]^{I}$ for $i=1, \ldots, k$. Thus, we see that $\left(S_{I}, s_{I}\right)$ is a local model of $X_{\mathrm{qs}}$ at $x$ and, after passing to the completion, $\xi_{i}$ (resp. $u_{j}$ ) is identified with $\chi^{e_{i}^{*}}\left(\operatorname{resp} . \chi^{\tilde{e}_{j}^{*}}\right)$ for $i=1, \ldots, k$ (resp. $\left.j=1, \ldots, n-k-1\right)$.

Let

$$
\omega_{x}=\frac{d \xi_{1}^{r_{I}^{\prime}} \wedge d\left(\xi_{2}^{c_{1}^{\prime}} / \xi_{1}^{c_{2}^{\prime}}\right) \wedge \cdots \wedge d\left(\xi_{k}^{c_{1}^{\prime}} / \xi_{1}^{c_{k}^{\prime}}\right) \wedge d u_{1} \wedge \cdots \wedge d u_{n-k-1}}{\xi_{1}^{r_{I}^{\prime}}\left(\xi_{2}^{c_{1}^{\prime}} / \xi_{1}^{c_{2}^{\prime}}\right) \cdots\left(\xi_{k}^{c_{1}^{\prime}} / \xi_{1}^{c_{k}^{\prime}}\right) u_{1} \cdots u_{n-k-1}}
$$

be the $(n-1)$-form on $X$, where $c_{i}^{\prime}$ and $r_{I}^{\prime}$ are defined in the same way as in case 2 . We see that $\omega_{x}$ can be identified with $\omega_{I}$ after passing to the completion and $\mathcal{M}_{x}^{[l]} \subset \mathbb{k}(X) \cdot \omega_{x}$, as in the case 1 . By Corollary 5.4, we have

$$
\mathbb{k}\left(S_{I}\right) \cdot \omega_{I}^{\otimes l} \cap\left(\Omega_{S_{I}}^{n-1}\right)^{[l]} \subset \mathcal{O}_{S_{I}} \cdot \chi^{l \alpha_{I}} \omega_{I}^{\otimes l},
$$

where, in this case, $\alpha_{I}=e_{1}^{*}+\cdots+e_{k}^{*}$. Put $h_{x}=\xi_{1}^{l} \cdots \xi_{k}^{l}$. Then, the function $h_{x}$ is identified with $\chi^{l \alpha_{I}}$ after passing to the completion.

Case 4: $I=\{n+1\}$.

After renumbering the indices of $a_{1}, \ldots, a_{n}$, we assume that Condition (2.3.2) holds for $j=1$ and $0=\operatorname{mult}_{p}\left(a_{1}\right)=\operatorname{mult}_{p}\left(a_{2}\right) \leq \operatorname{mult}_{p}\left(a_{3}\right) \leq$ $\cdots \leq \operatorname{mult}_{p}\left(a_{n}\right)$. In this case, we have $g_{I}=\xi_{0}$ and $f_{I}=f\left(\xi_{0}, \ldots, \xi_{n}\right)$. Thus, $\left(\partial\left(g_{I}-f_{I}\right) / \partial \xi_{0}\right)\left(P_{n+1}\right)=1-\left(\partial f_{I} / \partial \xi_{0}\right)\left(P_{n+1}\right) \neq 0$ and we can choose $\xi_{1}, \ldots, \xi_{n}$ as local coordinates of $C_{I}$ at $\tau_{I}^{-1}\left(P_{n+1}\right)$. Then, we see that $\left(S_{I}, s_{I}\right)$ is a local model of $X_{\mathrm{qs}}$ at $P_{n+1}$ and $\xi_{i}$ is identified with $\chi^{e_{i}^{*}}$ for $i=1, \ldots, n$ after passing to the completion. Let

$$
\omega_{x}=\frac{d\left(\xi_{2}^{a_{1}} / \xi_{1}^{a_{2}}\right) \wedge \cdots \wedge d\left(\xi_{n}^{a_{1}} / \xi_{1}^{a_{n}}\right)}{\left(\xi_{2}^{a_{1}} / \xi_{1}^{a_{2}}\right) \cdots\left(\xi_{n}^{a_{1}} / \xi_{1}^{a_{n}}\right)}
$$

be the rational $(n-1)$-form on $X$. We see that $\omega_{x}$ is identified with $\omega_{I}$ after passing to the completion and, as in case 1 , we have $\mathcal{M}_{P_{n+1}}^{[l]} \subset \mathbb{k}(X) \cdot \omega_{x}^{\otimes l}$. By Corollary 5.5, we have

$$
\mathbb{k}(S) \cdot \omega_{I}^{\otimes l} \cap\left(\Omega_{S_{I}}^{n-1}\right)^{[l]} \subset \mathcal{O}_{S_{I}} \cdot \chi^{l \alpha_{I}} \omega_{I}^{\otimes l} .
$$


Put $h_{x}=\xi_{1}^{l} \cdots \xi_{n}^{l}$. Then the function $h_{x}$ is identified with $\chi^{l \alpha_{I}}$ after passing to the completion.

Finally, let us prove (5). For $k=1, \ldots, r_{I}-1$, let

$$
\beta_{k}= \begin{cases}\left(1 / r_{I}\right)\left(\sum_{i=1}^{m}\left[k c_{i}\right]^{I} e_{i}+[k b]^{I} e_{n}\right) & \text { if } n+1 \notin I, \\ \left(1 / r_{I}\right) \sum_{i=1}^{m}\left[k c_{i}\right]^{I} e_{i} & \text { if } n+1 \in I \text { and } I \neq\{n+1\} \\ (1 / b) \sum_{i=1}^{n}\left[k c_{i}\right]^{I} e_{i} & \text { if } I=\{n+1\},\end{cases}
$$

be the lattice point of $\sigma_{I}$. We see that an exceptional divisor $E^{\prime}$ of $\varphi_{I}$ corresponds to some lattice point $\beta \in \sigma_{I} \cap \mathrm{N}_{I}$ which can be written as $\beta=\beta_{k}+\beta^{\prime}$ for some $k$ and some lattice point $\beta^{\prime}=\beta_{1}^{\prime} e_{1}+\cdots+\beta_{n}^{\prime} e_{n} \in \sigma_{I} \cap \mathrm{N}_{I}$, where $\beta_{i}^{\prime}$ is a nonnegative integer for $i=1, \ldots, n$.

Suppose that $I \neq\{n+1\}$. Then, we have

$$
\operatorname{mult}_{E^{\prime}}\left(\varphi_{I}^{*} \chi^{l \alpha_{I}}\right)=l \alpha_{I}(\beta) \geq l \alpha_{I}\left(\beta_{k}\right)=\left(l / r_{I}\right) \sum_{i \in I^{\#}}\left[k a_{i}\right]^{I}>l\left(r_{I}-A\right) / r_{I} .
$$

The last inequality follows from Condition (2.3.1). The rational $(n-1)$-form $\varphi_{I}^{*} \omega_{I}$ has a pole along $E^{\prime}$ with multiplicity one. Thus, the order of the pole of $\varphi_{I}^{*}\left(\chi^{l \alpha_{I}} \omega_{I}^{\otimes l}\right)$ along $E^{\prime}$ is $l-\operatorname{mult}_{E^{\prime}}\left(\varphi_{I}^{*} \chi^{\alpha_{I}}\right)<A l / r_{I}$.

Suppose that $I=\{n+1\}$. We assume that Condition (2.3.2) holds for $j=1$ and $0=\operatorname{mult}_{p}\left(a_{1}\right)=\operatorname{mult}_{p}\left(a_{2}\right) \leq \operatorname{mult}_{p}\left(a_{3}\right) \leq \cdots \leq \operatorname{mult}_{p}\left(a_{n}\right)$. If $\beta^{\prime} \neq 0$ then we have

$$
\operatorname{mult}_{E^{\prime}}\left(\varphi_{I}^{*} \chi^{l \alpha_{I}}\right)=l \alpha_{I}(\beta) \geq l \alpha_{I}\left(\beta^{\prime}\right)=l\left(\beta_{1}^{\prime}+\cdots+\beta_{n}^{\prime}\right) \geq l .
$$

Therefore, the order of the pole of $\varphi_{I}^{*}\left(\chi^{l \alpha_{I}} \omega_{I}^{\otimes l}\right)$ along $E^{\prime}$ is at most $l-$ $\operatorname{mult}_{p}\left(\varphi_{I}^{*} \chi^{\alpha_{I}}\right) \leq 0$. In the following, we assume that $\beta^{\prime}=0$, that is, $\beta=\beta_{k}$. If $k \notin \Psi_{1}$ then we can write $\left.\varphi_{I}^{*} \chi^{a_{1} e_{i}^{*}-a_{i} e_{1}^{*}}\right|_{U^{\prime}}=z^{p n_{i}} h_{i}$, where $U^{\prime}$ is an open set of $S^{\prime}, z$ is a defining equation for $E^{\prime}$ on $U^{\prime}, n_{i}$ is a nonnegative integer and $h_{i}$ is a holomorphic function which does not vanish along $E^{\prime}$. Hence, the form $\left.\varphi_{I}^{*} \omega_{I}\right|_{U^{\prime}}=\left(d h_{2} \wedge \cdots \wedge d h_{n}\right) /\left(h_{2} \cdots h_{n}\right)$ is holomorphic on $U^{\prime}$. If $k \in \Psi_{1}$ then we have

$$
\operatorname{mult}_{E^{\prime}}\left(\varphi_{I}^{*} \chi^{l \alpha_{I}}\right)=l \alpha_{I}(\beta)=l \alpha_{I}\left(\beta_{k}\right)=(l / b) \sum_{i=1}^{n}\left[k a_{i}\right]^{I}>l(b-A) / b .
$$

The last inequality follows from Condition (2.3.2). The $(n-1)$-form $\varphi_{I}^{*} \omega_{I}$ has a pole along $E^{\prime}$ with multiplicity one. Therefore, the order of the pole of $\varphi_{I}^{*}\left(\chi^{l \alpha_{I}} \omega_{I}^{\otimes l}\right)$ along $E^{\prime}$ is $l-\operatorname{mult}_{E^{\prime}}\left(\varphi_{I}^{*} \chi^{\alpha_{I}}\right)<A l / b$. This completes the proof. 
Next, consider a local model of $X_{\mathrm{qs}}$ at a point of $Z_{I}$ when $I$ is not saturated and $I \neq\{n+1\}$. In this case, there is a unique $I^{\prime} \subset\{1, \ldots, n+1\}$ such that $I^{\prime} \supset I, r_{I^{\prime}}=r_{I}$ and $I^{\prime}$ is saturated. Suppose that $S$ is the affine toric variety defined by $(\mathrm{N}, \sigma):=\left(\mathrm{N}_{I^{\prime}}, \sigma_{I^{\prime}}+\mathbb{R}_{\geq 0} \cdot \tilde{e}_{1}+\cdots+\mathbb{R}_{\geq 0} \cdot \tilde{e}_{i}\right)$ for some $i$. Since $\sigma_{I^{\prime}}$ is a face of $\sigma$, the toric variety $S_{I^{\prime}}$ can be seen as an open subvariety of $S$ and the rational $(n-1)$-form $\omega_{I^{\prime}}$ can be seen as a rational $(n-1)$-form on $S$.

LEMMA 5.8. Notation as above. Let $I$ be a subset of $\{1, \ldots, n+1\}$ such that $I$ is not saturated and $I \neq\{n+1\}$, and let $x \in Z_{I}$ be a point. Then, there is a nonnegative integer $n^{\prime}$ such that $\left(S_{I}, s_{I}\right)$ is a local model of $X_{\mathrm{qs}}$ at $x$, where $S_{I}$ is the affine toric variety defined by $\left(\mathrm{N}_{I}, \sigma_{I}\right):=\left(\mathrm{N}_{I^{\prime}}, \sigma_{I^{\prime}}+\right.$ $\left.\mathbb{R}_{\geq 0} \cdot \tilde{e}_{1}+\cdots+\mathbb{R}_{\geq 0} \cdot \tilde{e}_{n^{\prime}}\right)$ over $\mathbb{k}$ and $s_{I}$ is the distinguished point of $S_{I}$ which corresponds to the cone $\sigma_{I}$. Moreover, there is a rational $(n-1)$-form $\omega_{x}$ on $X$ and a function $h_{x} \in \mathcal{O}_{X, x}$ with the following properties.

(1) $\mathcal{M}_{x}^{[l]} \subset \mathbb{k}(X) \cdot \omega_{x}^{\otimes l}$.

(2) After passing to the completion, $\omega_{x}$ (resp. $\left.h_{x}\right)$ is identified with $\omega_{I}$ (resp. $\chi^{l \alpha_{I}}$ ) by the isomorphism $\hat{\mathcal{O}}_{X, x} \cong \hat{\mathcal{O}}_{S_{I}, s_{I}}$, where $\omega_{I}:=\omega_{I^{\prime}}$ and $\alpha_{I}:=\alpha_{I^{\prime}}$.

(3) We have $\mathbb{k}(S) \cdot \omega_{I}^{\otimes l} \cap\left(\Omega_{S_{I}}^{n-1}\right)^{[l]} \subset \mathcal{O}_{S_{I}} \cdot \chi^{l \alpha_{I}} \omega_{I}^{\otimes l}$.

(4) Let $\varphi_{I}: S^{\prime} \rightarrow S_{I}$ be a toric resolution obtained by subdividing the cone $\sigma_{I}$. Then, for any exceptional divisor $E^{\prime}$ of $\varphi_{I}$, the order of the pole of $\varphi_{I}^{*}\left(\chi^{l \alpha_{I}} \omega_{I}^{\otimes l}\right)$ along $E^{\prime}$ is at most $A l / r_{I}-1$.

Proof. Put $k=|I|$. Consider the affine variety

$$
U_{I}:=\operatorname{Spec} \mathbb{k}\left[\left\{\xi_{i} \mid i \in\{0, \ldots, n+1\} \backslash I\right\}\right] \times \operatorname{Spec} \mathbb{k}\left[u_{1}, u_{1}^{-1}, \ldots, u_{k-1}, u_{k-1}^{-1}\right]
$$

on which $\boldsymbol{\mu}_{r_{I}}$ acts and gives the quotient $\left(U_{I} / \boldsymbol{\mu}_{r_{I}}\right) \cong \mathrm{D}_{+}\left(x_{I}\right)$. Then, $U_{I^{\prime}}$ is the open subvariety $D\left(\prod_{i \in I^{\prime} \backslash I} \xi_{i}\right)$ of $U_{I}$. We have $f_{I}=f^{\prime}+\sum_{i \in I^{\prime} \backslash I} f_{i}^{\prime} \xi_{i}+f^{\prime \prime}$, where $f^{\prime}, f_{i}^{\prime}$ are functions of $u_{1}, \ldots, u_{k-1}$ and $f^{\prime \prime} \in\left(\left\{\xi_{i} \mid i \in\{0, \ldots, n+\right.\right.$ $1\} \backslash I\})^{2}$. By Lemma 3.6, we see that there is some $i \in I^{\prime} \backslash I$ such that $\left(\partial\left(g_{I}-f_{I}\right) / \partial \xi_{i}\right)(x) \neq 0$, or there is some $j \in\{1, \ldots, k-1\}$ such that $\left(\partial\left(g_{I}-f_{I}\right) / \partial u_{j}\right)(x) \neq 0$. In the former case, let $x^{\prime}$ be a point of $Z_{I^{\prime}}$ such that $\left(\partial\left(g_{I}-f_{I}\right) / \partial \xi_{i}\right)\left(x^{\prime}\right) \neq 0$ and in the latter case, let $x^{\prime}$ be a point of $Z_{I^{\prime}}$ such that $\left(\partial\left(g_{I}-f_{I}\right) / \partial u_{j}\right)\left(x^{\prime}\right) \neq 0$. Then, by the proof of Lemma 5.7 , we see that $\left(S_{I^{\prime}}, s_{I^{\prime}}\right)$ is a local model of $X_{\mathrm{qs}}$ at $x^{\prime}$ and we may assume that $\xi_{i}$ (resp. $u_{j}$ ) is identified with $\chi_{i}^{e_{i}^{*}}\left(\operatorname{resp} . \chi^{\tilde{e}_{j}^{*}}\right)$. Moreover, there is a nonnegative 
integer $n^{\prime}$ such that $\left(S_{I}, s_{I}\right)$ is a local model of $X_{\mathrm{qs}}$ at $x$, where $S_{I}$ is the affine variety defined by $\left(\mathrm{N}_{I}, \sigma_{I}\right):=\left(\mathrm{N}_{I^{\prime}}, \sigma_{I^{\prime}}+\mathbb{R}_{\geq 0} \cdot \tilde{e}_{1}+\cdots \mathbb{R}_{\geq 0} \cdot \tilde{e}_{n^{\prime}}\right)$ and $s_{I}$ is the distinguished point of $S_{I}$ which corresponds to the cone $\sigma_{I}$.

Put $\omega_{I}:=\omega_{I^{\prime}}$ and $\alpha_{I}:=\alpha_{I^{\prime}}$. As in the proof of Lemma 5.7, we can write down explicitly the rational $(n-1)$-form $\omega_{x}$ on $X$ and the function $h_{x} \in \mathcal{O}_{X, x}$ with the properties (1) and (2). By Corollary 5.4, we see that $\mathbb{k}\left(S_{I}\right) \cdot \omega_{I}^{\otimes l} \cap\left(\Omega_{S_{I}}^{n-1}\right)^{[l]} \subset \mathcal{O}_{S_{I}} \cdot \chi^{l \alpha_{I}} \omega_{I}^{\otimes l}$.

Let us prove (4). For $k=1, \ldots, r_{I}-1$, let

$$
\beta_{k}= \begin{cases}\left(1 / r_{I}\right)\left(\sum_{i=1}^{m}\left[k c_{i}\right]^{I} e_{i}+[k b]^{I} e_{n}\right) & \text { if } n+1 \notin I^{\prime}, \\ \left(1 / r_{I}\right) \sum_{i=1}^{m}\left[k c_{i}\right]^{I} e_{i} & \text { if } n+1 \in I^{\prime},\end{cases}
$$

be lattice points of $\sigma_{I}$. The exceptional divisor $E^{\prime}$ corresponds to some lattice point $\beta \in \sigma_{I} \cap \mathrm{N}_{I}$ which can be written as $\beta=\beta_{k}+\beta^{\prime}$ for some $k$ and some lattice point $\beta^{\prime} \in \sigma_{I} \cap \mathrm{N}_{I}$. The $(n-1)$-form $\varphi_{I}^{*} \omega_{I}$ has a pole along $E^{\prime}$ with multiplicity one. Hence, the order of the pole of $\varphi_{I}^{*}\left(\chi^{l \alpha_{I}} \omega_{I}^{\otimes l}\right)$ along $E^{\prime}$ is $l-\operatorname{mult}_{E^{\prime}}\left(\varphi_{I}^{*} \chi^{l \alpha_{I}}\right)$. We have

$$
\operatorname{mult}_{E^{\prime}}\left(\varphi_{I}^{*} \chi^{l \alpha_{I}}\right)=l \alpha_{I}(\beta) \geq l \alpha_{I}\left(\beta_{k}\right)=\left(l / r_{I}\right) \sum_{i \in I^{\prime \#}}\left[k a_{i}\right]>l\left(r_{I}-A\right) / r_{I} .
$$

The last inequality follows from Condition (2.3.1) for $I^{\prime}$. Therefore, the lemma is proved.

Proof of Lemma 4.8. We may assume that $r_{I}>1$, that is, $Z_{I}$ is contained in the singular locus of $X$. Indeed, if $r_{I}=1$ then $Z_{I}$ is contained in the smooth locus of $X$. Hence, generically around $Z_{I}$, the invertible sheaf $\mathcal{M}^{[l]}$ is generated by a holomorphic form since $\mathcal{M}^{[l]} \subset\left(\Omega_{X}^{n-1}\right)^{[l]}$. By the definition of $\varepsilon_{i}$, we see that $\varepsilon_{i} \leq 0$. On the other hand, we have $A>0$ by Condition (2.1.4).

Let $x \in Z_{I}$ be any point. Let $\left(S_{I}, s_{I}\right)$ be the local model of $X_{\mathrm{qs}}$ at $x, \omega_{I}$ the rational $(n-1)$-form on $S_{I}$ and $\alpha_{I}$ the lattice point of $\sigma_{I}^{\vee} \cap \mathrm{M}_{I}$ which are defined in Definition 5.6 or Lemma 5.8. By Lemma 5.7 and 5.8, there are a function $h_{x} \in \mathcal{O}_{X, x}$ and a rational $(n-1)$-form $\omega_{x}$ such that $\mathcal{M}_{x}^{[l]} \subset \mathcal{O}_{X, x} \cdot h_{x} \omega_{x}^{\otimes l}$ and, after passing to the completion, $h_{x} \omega_{x}^{\otimes l}$ is identified with $\chi^{l \alpha_{I}} \omega_{I}^{\otimes l}$. Let $\varphi^{\prime}: S^{\prime} \rightarrow S_{I}$ be the resolution of $S_{I}$ which is induced by the resolution $\varphi$ of the toroidal embedding $X_{\mathrm{qs}}$ and let $E^{\prime}$ be the exceptional divisor of $\varphi^{\prime}$ which corresponds formally to $E_{i}$. By Lemma 5.7 or Lemma 5.8, we see that the order of the pole of $\varphi^{*}\left(h_{x} \omega_{x}^{\otimes l}\right)$ along $E_{i}$ coincides with that of $\varphi^{\prime *}\left(\chi^{\alpha_{I}} \omega_{I}^{\otimes l}\right)$ along $E^{\prime}$ and is at most $A l / r_{I}-1$. Therefore, we have $A l / r_{I}>l \varepsilon_{i}$ and the lemma is proved. 


\section{§6. Proof of main theorems}

Proof of Theorem 1.3. This follows from Lemma 4.5, 4.9 and 1.6.

Proof of Theorem 1.2. Theorem 1.3 and 1.5 imply that the weighted hypersurface $X_{f}$ defined over $\mathbb{C}$ is not ruled for a very general $f \in H_{d}(\mathbb{C})$ (cf. [12, Section 4.4]). If $X=X_{f}$ is defined over $\mathbb{C}$, it is quasi smooth for a general $f \in H_{d}(\mathbb{C})$ by Corollary 3.7. Therefore, $X$ has only quotient singularities for a general $f \in H_{d}(\mathbb{C})$. We have

$$
\mathcal{O}_{X}\left(-K_{X}\right) \cong \mathcal{O}_{X}\left(\sum_{i=0}^{n} a_{i}+b-d\right) \text {. }
$$

Thus, Condition (2.1.4) implies that $-K_{X}$ is ample. This completes the proof.

\section{$\S 7$. Examples of nonrational $\mathbb{Q}$-Fano varieties}

In this section, we present some examples of nonrational $\mathbb{Q}$-Fano varieties. In Theorem 7.1 and 7.3 below, we do not prove that $\left(p,\left\{a_{i}\right\}, b, n, d\right)$ satisfies Condition (2.1.5). This is because there are computer programs for checking quasi smoothness and our cases can be done easily, or one can also prove it directly.

\subsection{Nonrational terminal $\mathbb{Q}$-Fano threefolds}

There are lists $[7,16.6]$, [2, Table 1] and [3, Table 1] of weighted hypersurfaces of dimension three which are terminal $\mathbb{Q}$-Fano varieties. We obtained Table 1 below by choosing members of those lists that satisfy both Condition 2.1 and 2.3.

Let $\left(p,\left\{a_{i}\right\}, b, n, d\right)$ be one of those listed in Table 1. It is straightforward to check that it satisfies Condition 2.1 and 2.3. The integer $c$ in Table 1 is defined as $c:=-d+\sum_{i=0}^{n} a_{i}>0$ so that we have $\mathcal{O}_{X}\left(-K_{X}\right) \cong \mathcal{O}_{X}(c)$. The singular points of $X$ and types of singularities of $X$ are written in the last column. $P_{i}$ stands for the point $(0: \cdots: 0: 1: 0: \cdots: 0)$, where the 1 is in the $i$-th position, and $P_{i j}$ is a point contained in the singular stratum $Z_{\{i, j\}}$. As a result, we obtain the following examples.

ThEOREM 7.1. Let $p, a_{0}, \ldots, a_{3}, b$ and $d$ be integers listed in Table 1. Then, the weighted hypersurface

$$
X_{f}:=\left(y^{p} x_{0}-f\left(x_{0}, \ldots, x_{3}\right)=0\right) \subset \mathbb{P}_{\mathbb{C}}\left(1, a_{1}, a_{2}, a_{3}, b\right)
$$

is a non-ruled terminal $\mathbb{Q}$-Fano threefold for a very general $f \in H_{d}(\mathbb{C})$. 
Table 1: A List of $\left(p,\left\{a_{i}\right\}, b, n, d\right)$ satisfying Condition 2.1 and 2.3.

\begin{tabular}{|c||c|c|c|c|c|}
\hline & $d$ & $p$ & $\left(a_{0}, \ldots, a_{3}, b\right)$ & $c$ & Singularities \\
\hline No. 1 & 5 & 2 & $(1,1,1,1,2)$ & 1 & $P_{4}: \frac{1}{2}(1,1,1)$ \\
No. 2 & 7 & 2 & $(1,1,1,2,3)$ & 1 & $P_{3}: \frac{1}{2}(1,1,1), P_{4}: \frac{1}{3}(1,1,2)$ \\
No. 3 & 9 & 2 & $(1,1,1,3,4)$ & 1 & $P_{4}: \frac{1}{4}(1,1,3)$ \\
No. 4 & 10 & 3 & $(1,1,1,5,3)$ & 1 & $P_{4}: \frac{1}{3}(1,1,2)$ \\
No. 5 & 10 & 3 & $(1,1,2,5,3)$ & 2 & $P_{4}: \frac{1}{3}(1,1,2)$ \\
No. 6 & 15 & 2 & $(1,1,2,5,7)$ & 1 & $P_{2}: \frac{1}{2}(1,1,1), P_{4}: \frac{1}{7}(1,2,5)$ \\
No. 7 & 15 & 2 & $(1,1,3,4,7)$ & 1 & $P_{3}: \frac{1}{4}(1,1,3), P_{4}: \frac{1}{7}(1,3,4)$ \\
No. 8 & 15 & 2 & $(1,2,3,5,7)$ & 3 & $P_{1}: \frac{1}{2}(1,1,1), P_{4}: \frac{1}{7}(1,3,4)$ \\
No. 9 & 16 & 3 & $(1,1,2,8,5)$ & 1 & $P_{23}: \frac{1}{2}(1,1,1), P_{4}: \frac{1}{5}(1,2,3)$ \\
No. 10 & 21 & 2 & $(1,1,3,7,10)$ & 1 & $P_{4}: \frac{1}{10}(1,3,7)$ \\
No. 11 & 22 & 3 & $(1,1,3,11,7)$ & 1 & $P_{2}: \frac{1}{3}(1,1,2), P_{4}: \frac{1}{7}(1,3,4)$ \\
No. 12 & 28 & 3 & $(1,1,4,14,9)$ & 1 & $P_{23}: \frac{1}{2}(1,1,1), P_{4}: \frac{1}{9}(1,4,5)$ \\
\hline
\end{tabular}

Remark 7.2. [7, 16.6] (resp. [3, Table 1], [2, Table 1]) is the list of terminal $\mathbb{Q}$-Fano weighted hypersurfaces of dimension three with $c=1$ (resp. $c=2, c \geq 3$ ). We remark that $[7,16.6]$ is the complete list while $[3$, Table 1] and [2, Table 1] may not be complete lists.

It is proved in [5] that a general member of each of the 95 families listed in $[7,16.6]$ are (birationally) rigid, which implies the nonrationality of the general member. Thus, among the twelve families of our examples, No. 5 and 8 are new and the remaining ten provide the known cases with an alternate proof of nonrationality. Nevertheless, our examples are new in the sense that our proof shows that all unirulings have degree divisible by 2 (if $p=2$ ) or 3 (if $p=3$ ).

\subsection{Nonrational $\log$ terminal $\mathbb{Q}$-Fano varieties of dimension $\geq 4$}

Let $m, n$ be integers such that $4 \leq n, 0<m<n$ and let $l$ be an odd integer such that $n-m+1<l<2(n-m)$. Then, for every odd positive integer $a$ with $a>(m+1) / 2$, the combination

$$
\left(p, a_{0}, \ldots, a_{m}, a_{m+1}, \ldots, a_{n}, b, n, d\right)=(2,1, \ldots, 1, a, \ldots, a,(a l-1) / 2, n, a l)
$$

satisfies Condition 2.1. We show that $\left(p,\left\{a_{i}\right\}, b, n, d\right)$ satisfies Condition 2.3 for every odd positive integer $a$. We see that $\mathcal{I}_{\text {sat }}=\left\{I_{1}, I_{2}\right\}$, where $I_{1}=$ 
$\{m+1, \ldots, n\}$ and $I_{2}=\{n+1\}$. Thus, $\left(p,\left\{a_{i}\right\}, b, n, d\right)$ satisfies Condition (2.3.1) since we have

$$
A:=d-\sum_{i=0}^{n} a_{i}=(l+m-n) a-(m+1)
$$

and

$$
\min _{0<k<a}\left\{\sum_{i=0}^{m}\left[k a_{i}\right]^{I_{1}}\right\}=m+1
$$

Moreover, $\left(p,\left\{a_{i}\right\}, b, n, d\right)$ satisfies Condition (2.3.2) for $j=1$. Indeed, we see that $i l \notin \Psi_{1}$ for $i=1, \ldots,(a-1) / 2$ and, for an integer $c$ with $0<c \leq(a-1) / 2,[k a]^{I}=c$ if and only if $k \equiv c l(\bmod b)$. In other words, we have $[k a]^{I} \geq(a+1) / 2$ for $k \in \Psi_{1}$ and hence

$$
\begin{aligned}
\min _{k \in \Psi_{1}}\left\{\sum_{i=1}^{n}\left[k a_{i}\right]^{I}\right\} & =\min _{k \in \Psi_{1}}\left\{m[k]^{I}+(n-m)[k a]^{I}\right\} \\
& \geq m+(n-m)(a+1) / 2 .
\end{aligned}
$$

We have

$$
\begin{aligned}
A & -\left(b-\min _{k \in \Psi_{1}}\left\{\sum_{i=1}^{n}\left[k a_{i}\right]^{I}\right\}\right) \\
& \geq(l+m-n) a-(m+1)-(a l-1) / 2+m+(n-m)(a+1) / 2 \\
& =((l+m-n) a+n-m-1) / 2>0
\end{aligned}
$$

since $l+m-n>0$ and $n-m>0$. Therefore, $\left(p,\left\{a_{i}\right\}, b, n, d\right)$ satisfies Condition (2.3.2) and we obtain the following examples.

THEOREM 7.3. Let $m, n$ be integers such that $4 \leq n$ and $0<m<n$, and let $l$ be an odd positive integer such that $n-m+1<l<2(n-m)$. Then, for every odd positive integer a with $a>(m+1) / 2$, the weighted hypersurface

$$
X_{f}:=\left(y^{2} x_{0}-f\left(x_{0}, \ldots, x_{n}\right)=0\right) \subset \mathbb{P}_{\mathbb{C}}\left(1^{m+1}, a^{n-m},(a l-1) / 2\right)
$$

of degree al is a non-ruled log terminal $\mathbb{Q}$-Fano variety for a very general $f\left(x_{0}, \ldots, x_{n}\right) \in H_{a l}(\mathbb{C})$.

Here, $\mathbb{P}\left(1^{m+1}, a^{n-m},(a l-1) / 2\right)$ is the weighted projective space

$$
\mathbb{P}(\overbrace{1, \ldots, 1}^{m+1}, \overbrace{a, \ldots, a}^{n-m},(a l-1) / 2) .
$$


Remark 7.4. The singular locus of $X_{f}$ is the union of $\bar{Z}_{I_{1}}=\left(x_{0}=\right.$ $\left.\cdots=x_{m}=y=0\right) \cap X_{f}$ and $Z_{I_{2}}=\left\{P_{n+1}\right\}$. The singularity of $X_{f}$ at each point of $\bar{Z}_{I_{1}}$ is of type

$$
\frac{1}{a}(\overbrace{1, \ldots, 1}^{m+1}, \overbrace{0, \ldots, 0}^{n-m-2}, b)=\frac{1}{a}(\overbrace{2, \ldots, 2}^{m+1}, \overbrace{0, \ldots, 0}^{n-m-2},-1)
$$

and that of $X_{f}$ at $P_{n+1}$ is of type

$$
\frac{1}{b}(\overbrace{1, \ldots, 1}^{m}, \overbrace{a, \ldots, a}^{n-m})=\frac{1}{b}(\overbrace{l, \ldots, l}^{m}, \overbrace{1, \ldots, 1}^{n-m}),
$$

where $b=(a l-1) / 2$.

\section{REFERENCES}

[1] M. Artin and D. Mumford, Some elementary examples of uniruled varieties which are not rational, Proc. London. Math. Soc., 25 (1972), 75-95.

[2] G. Brown and K. Suzuki, Computing certain Fano 3-folds, Japan J. Indust. Appl. Math., 24 (2007), 241-250.

[3] G. Brown and K. Suzuki, Fano 3-folds with divisible anticanonical class, Manuscripta Math., 123 (2007), 37-51.

[4] H. Clemens and P. Griffiths, The intermediate Jacobian of the cubic threefold, Ann. of Math. (2), 95 (1972), 281-356.

[5] A. Corti, A. Pukhlikov and M. Reid, Fano 3-fold hypersurfaces, Explicit birational geometry of 3-folds, Cambridge Univ. Press, 2000, pp. 175-258.

[6] I. Dolgachev, Weighted projective spaces, Group actions and vector fields, Proc. Vancouver (1981), LNM 956, Springer Verlag, pp. 34-71.

[7] A. R. Iano-Fletcher, Working with weighted complete intersections, Explicit birational geometry of 3-folds, Cambridge Univ. Press, 2000, pp. 101-173.

[8] V. A. Iskovskikh and Ju. I. Manin, Three-dimensional quartics and counterexamples to the Lüroth problem, Math. USSR-Sb., 15 (1971), 815-868.

[9] G. Kempf, F. Knudsen, D. Mumford and B. Saint-Donat, Toroidal embeddings I, LNM 339, Springer Verlag, 1973.

[10] J. Kollár, Nonrational hypersurfaces, J. AMS, 8 (1995), 241-249.

[11] J. Kollár, Rational curves on algebraic varieties, Ergebnisse der Math. vol. 32, Springer Verlag, 1996.

[12] J. Kollár, K. E. Smith and A. Corti, Rational and nearly rational varieties, Cambridge studies in advanced mathematics 92, Cambridge Univ. Press, 2004.

[13] T. Matsusaka, Algebraic deformations of polarized varieties, Nagoya Math. J., 31 (1968), 185-245.

[14] A. V. Pukhlikov, Birational automorphisms of Fano hypersurfaces, Invent. Math., 134 (1998), no. 2, 401-426. 
[15] Q. Zhang, Rational connectedness of $\log \mathbb{Q}$-Fano varieties, J. Reine Angew. Math., 590 (2006), 131-142.

Research Institute for Mathematical Sciences

Kyoto University

Kyoto 606-8502

Japan

takuzo@kurims.kyoto-u.ac.jp 